\title{
The Cauchy problem for a strongly degenerate quasilinear equation
}

Received 15 April 2004 and in revised form 20 October 2004

\begin{abstract}
We prove existence and uniqueness of entropy solutions for the Cauchy problem for the quasilinear parabolic equation $u_{t}=\operatorname{div} \mathbf{a}(u, D u)$, where $\mathbf{a}(z, \xi)=\nabla_{\xi} f(z, \xi)$, and $f$ is a convex function of $\xi$ with linear growth as $\|\xi\| \rightarrow \infty$, satisfying other additional assumptions. In particular, this class includes a relativistic heat equation and a flux limited diffusion equation used in the theory of radiation hydrodynamics.
\end{abstract}

\section{Introduction}

We are interested in the problem

$$
\begin{cases}\frac{\partial u}{\partial t}=\operatorname{div} \mathbf{a}(u, D u) & \text { in } Q_{T}=(0, T) \times \mathbb{R}^{N}, \\ u(0, x)=u_{0}(x) & \text { in } x \in \mathbb{R}^{N},\end{cases}
$$

where $0 \leq u_{0} \in L^{1}\left(\mathbb{R}^{N}\right) \cap L^{\infty}\left(\mathbb{R}^{N}\right), \mathbf{a}(z, \xi)=\nabla_{\xi} f(z, \xi)$ and $f$ is a function with linear growth as $\|\xi\| \rightarrow \infty$.

Particular instances of problem [1.1) have been studied in [12] and [19], when $N=1$. In these papers the authors considered the problem

$$
\begin{cases}\frac{\partial u}{\partial t}=\left(\varphi(u) \mathbf{b}\left(u_{x}\right)\right)_{x} & \text { in }(0, T) \times \mathbb{R} \\ u(0, x)=u_{0}(x) & \text { in } x \in \mathbb{R}\end{cases}
$$

corresponding to $\sqrt{1.1}$ when $N=1$ and $\mathbf{a}\left(u, u_{x}\right)=\varphi(u) \mathbf{b}\left(u_{x}\right)$, where $\varphi: \mathbb{R} \rightarrow \mathbb{R}^{+}$ is smooth and strictly positive, and $\mathbf{b}: \mathbb{R} \rightarrow \mathbb{R}$ is a smooth odd function such that $\mathbf{b}^{\prime}>0$ and $\lim _{s \rightarrow \infty} \mathbf{b}(s)=\mathbf{b}_{\infty}$. Such models appear in the theory of phase transitions where the corresponding free energy functional has a linear growth rate with respect to the gradient [26]. As the authors observed, in general, there are no classical solutions of [1.1); they defined the notion of entropy solution and proved existence ([12]) and uniqueness ([19]) of entropy solutions of [1.1]. Existence was proved for bounded strictly increasing

F. Andreu, J. M. Mazón: Departamento de Análisis Matemático, Universitat de Valencia, Dr. Moliner 50, 45100 Burjassot (Valencia), Spain; e-mail: Fuensanta.Andreu@uv.es, Mazon@uv.es

V. Caselles: Departamento de Tecnologia, Universitat Pompeu-Fabra, Passeig de Circumvalacró 8, 08003 Barcelona, Spain; e-mail: Vicent.Caselles@upf.edu 
initial conditions $u_{0}: \mathbb{R} \rightarrow \mathbb{R}$ such that $\mathbf{b}\left(u_{0}^{\prime}\right) \in C(\mathbb{R})$ (where $\mathbf{b}\left(u_{0}^{\prime}\left(x_{0}\right)\right)=\mathbf{b}_{\infty}$ if $u_{0}$ is discontinuous at $\left.x_{0}\right)$ and $\mathbf{b}\left(u_{0}^{\prime}(x)\right) \rightarrow 0$ as $x \rightarrow \pm \infty$ [12]. The entropy condition was written in Oleĭnik's form and uniqueness was proved using suitable test functions constructed by regularizing the sign of the difference of two solutions.

In [13], Blanc considered the following Neumann problem in an interval of $\mathbb{R}$ :

$$
\begin{cases}\frac{\partial u}{\partial t}=\left(\mathbf{a}\left(u, u_{x}\right)\right)_{x} & \text { in }(0, T) \times(0,1), \\ u_{x}(t, 0)=u_{x}(t, 1)=0 & \text { in } t \in(0, T), \\ u(0, x)=u_{0}(x) & \text { in } x \in(0,1),\end{cases}
$$

where $\mathbf{a}(u, v)$ is a function of class $C^{1, \alpha}([0, \infty) \times \mathbb{R})$ satisfying other additional assumptions. He associated an $m$-accretive operator to $-\left(\mathbf{a}\left(u, u_{x}\right)\right)_{x}$ with Neumann boundary conditions, and proved the existence and uniqueness of a semigroup solution of (1.3). An example of the equations considered in [13] is the so called plasma equation (see [22])

$$
\frac{\partial u}{\partial t}=\left(\frac{u^{5 / 2} u_{x}}{1+u\left|u_{x}\right|}\right)_{x} \quad \text { in }(0, T) \times(0,1),
$$

where the initial condition $u_{0}$ is assumed to be positive. In this case $u$ represents the temperature of electrons, and the form of the conductivity $\mathbf{a}\left(u, u_{x}\right)=u^{5 / 2} u_{x} /\left(1+u\left|u_{x}\right|\right)$ has the effect of limiting the heat flux. But, as far as we know, existence and uniqueness results for higher dimensional problems have not been considered in the literature. This was the purpose of our papers [4] and [5] in which we studied the Neumann problem for Lagrangians $f$ satisfying the following coercivity and linear growth condition:

$$
C_{0}\|\xi\|-D_{0} \leq f(z, \xi) \leq M_{0}(1+\|\xi\|)
$$

for some positive constants $C_{0}, M_{0}$. Now, there are some relevant cases like the relativistic heat equation (see [14], [27])

$$
u_{t}=v \operatorname{div}\left(\frac{|u| D u}{\sqrt{u^{2}+a^{2}|D u|^{2}}}\right)
$$

for which the Lagrangian $f(z, \xi)=\left(v / a^{2}\right)|z| \sqrt{z^{2}+a^{2}|\xi|^{2}}$ does not satisfy $[1.5)$. Observe that, in this case, $f(z, \xi)$ satisfies the following condition:

$$
C_{0}(z)\|\xi\|-D_{0}(z) \leq f(z, \xi) \leq M_{0}(z)(\|\xi\|+1)
$$

for any $(z, \xi) \in \mathbb{R} \times \mathbb{R}^{N}$, and some positive and continuous functions $C_{0}, D_{0}, M_{0}$ such that $C_{0}(z)>0$ for any $z \neq 0$. The equation 1.6 was introduced by $\mathrm{Ph}$. Rosenau in [27] to overcome the unphysical dependence of the flux on the gradient as predicted by the classical transport theory. He imposed the acoustic speed as an upper bound of the permitted propagation speed in a medium. This provides the means to control the growth of the flux; flux saturates as the gradients become unbounded. Let us also mention that equation (1.6) was recently derived by Y. Brenier by means of Monge-Kantorovich's mass transport theory $([14])$. As Brenier pointed out in [14], this relativistic heat equation 
is one among the various flux limited diffusion equations used in the theory of radiation hydrodynamics [25]. Indeed, a very similar equation

$$
u_{t}=v \operatorname{div}\left(\frac{u D u}{u+\frac{v}{c}|D u|}\right)
$$

can be found in [25].

The purpose of the present paper is to extend the results in [4] and [5] to the case where the Lagrangian $f$ satisfies 1.7 and the initial condition is in $\left(L^{1}\left(\mathbb{R}^{N}\right) \cap L^{\infty}\left(\mathbb{R}^{N}\right)\right)^{+}$.

In [6] we have considered the elliptic problem

$$
u-\operatorname{div} \mathbf{a}(u, D u)=v \quad \text { in } \mathbb{R}^{N} .
$$

By introducing a notion of entropy solution for $[1.9]$ we proved in [6] the existence and uniqueness of an entropy solution of 1.9 when $v \in\left(L^{1}\left(\mathbb{R}^{N}\right) \cap L^{\infty}\left(\mathbb{R}^{N}\right)\right)^{+}$. This permits us to define an accretive operator $B$ in $L^{1}\left(\mathbb{R}^{N}\right)$ whose domain is contained in $\left(L^{1}\left(\mathbb{R}^{N}\right) \cap L^{\infty}\left(\mathbb{R}^{N}\right)\right)^{+}$(which amounts to considering the right hand side $v$ of 1.9 in $\left.\left(L^{1}\left(\mathbb{R}^{N}\right) \cap L^{\infty}\left(\mathbb{R}^{N}\right)\right)^{+}\right)$and whose closure $\mathcal{B}$ is $\mathrm{m}$-accretive (hence, it generates a nonlinear contraction semigroup $T(t))$ in $L^{1}\left(\mathbb{R}^{N}\right)^{+}([11]$, [17]). However, we have not been able to characterize $\mathcal{B}$ in distributional terms. In spite of this, the knowledge of the operator $B$ and the fact that, if $u$ is the entropy solution of [1.9), then $\|u\|_{\infty} \leq\|v\|_{\infty}$, permit us to use Crandall-Ligget's iteration scheme and define

$$
u(t):=T(t) u_{0}=\lim _{n \rightarrow \infty}\left(I+\frac{t}{n} B\right)^{-n} u_{0}, \quad u_{0} \in\left(L^{1}\left(\mathbb{R}^{N}\right) \cap L^{\infty}\left(\mathbb{R}^{N}\right)\right)^{+} .
$$

The main purpose of this paper is to prove that $u(t)$ is an entropy solution of 1.1 (a notion that will be defined in Section 4), and that entropy solutions are unique. As a technical tool we shall use some lower semicontinuity results (see [18] and [20]) for energy functionals whose density is a function $g(x, u, D u)$ convex in $D u$ with linear growth rate as $|D u| \rightarrow \infty$. The qualitative behavior of solutions of (1.6) and the motion of its support will be the object of a subsequent paper [7].

Finally, let us explain the plan of the paper. In Section 2 we recall some basic facts about function spaces, functions of bounded variation, denoted by BV $(\Omega)$, Green's formula, and lower semicontinuity results for energy functionals defined in $\mathrm{BV}(\Omega)$. In Section 3 we state the main assumptions on the Lagrangian $f$, recall the meaning of expressions of type $f(u, D u)$ for functions $u$ in $\mathrm{BV}\left(\mathbb{R}^{N}\right)$ and define an associated functional calculus. We also recall the notion of entropy solution for the elliptic problem (1.9) and the existence and uniqueness results for it proved in [6]. Then we translate this result into the language of accretive operators to be able to apply Crandall-Liggett's iteration scheme to prove existence of solutions of 1.1 for initial data $u_{0} \in L^{1}\left(\mathbb{R}^{N}\right) \cap L^{\infty}\left(\mathbb{R}^{N}\right), u_{0} \geq 0$. This will be the main purpose of Section 4 where we define the notion of entropy solution of (1.1) and we prove that Crandall-Liggett's iteration scheme produces entropy solutions of it. Then we prove uniqueness of entropy solutions by using Kruzhkov's doubling variables technique. 


\section{Preliminaries}

\subsection{Some function spaces. BV functions}

Let us start with some notation. We denote by $\mathcal{L}^{N}$ and $\mathcal{H}^{N-1}$ the $N$-dimensional Lebesgue measure and the $(N-1)$-dimensional Hausdorff measure in $\mathbb{R}^{N}$, respectively. Given an open set $\Omega$ in $\mathbb{R}^{N}$ we shall denote by $\mathcal{D}(\Omega)$, or $C_{0}^{\infty}\left(\mathbb{R}^{N}\right)$, the space of infinitely differentiable functions with compact support in $\Omega$. The space of continuous functions with compact support in $\mathbb{R}^{N}$ will be denoted by $C_{c}\left(\mathbb{R}^{N}\right)$.

We shall use several notations borrowed from [10]. Let $M\left(\mathbb{R}^{N}\right)$ be the set of Lebesgue measurable functions from $\mathbb{R}^{N}$ into $\mathbb{R}$. We denote by $L\left(\mathbb{R}^{N}\right)$ the space $L\left(\mathbb{R}^{N}\right):=L^{1}\left(\mathbb{R}^{N}\right)$ $+L^{\infty}\left(\mathbb{R}^{N}\right)$, which equipped with the norm

$$
\|u\|_{1+\infty}:=\inf \left\{\left\|u_{1}\right\|_{1}+\left\|u_{2}\right\|_{\infty}: u=u_{1}+u_{2}, u_{1} \in L^{1}\left(\mathbb{R}^{N}\right), u_{2} \in L^{\infty}\left(\mathbb{R}^{N}\right)\right\}
$$

is a Banach space. If we set

$$
L_{0}\left(\mathbb{R}^{N}\right):=\left\{u \in M\left(\mathbb{R}^{N}\right): \int_{\mathbb{R}^{N}}(|u|-k)^{+}<\infty \forall k>0\right\},
$$

we have $L_{0}\left(\mathbb{R}^{N}\right)={\overline{L^{1}\left(\mathbb{R}^{N}\right) \cap L^{\infty}\left(\mathbb{R}^{N}\right)}}^{\|\|_{1+\infty}}\left([[10])\right.$. The dual space of $L_{0}\left(\mathbb{R}^{N}\right)$ is isometrically isomorphic to $L^{1 \cap \infty}\left(\mathbb{R}^{N}\right):=L^{1}\left(\mathbb{R}^{N}\right) \cap L^{\infty}\left(\mathbb{R}^{N}\right)$, when $L^{1 \cap \infty}\left(\mathbb{R}^{N}\right)$ is endowed with the norm $\|u\|_{1 \cap \infty}:=\max \left\{\|u\|_{1},\|u\|_{\infty}\right\}([10])$.

Given $u, v \in M\left(\mathbb{R}^{N}\right)$, we shall write

$$
u<<v \text { if and only if } \int_{\mathbb{R}^{N}} j(u) d x \leq \int_{\mathbb{R}^{N}} j(v) d x
$$

for all $j \in J_{0}:=\{j: \mathbb{R} \rightarrow[0, \infty]$, convex, 1.s.c., $j(0)=0\}$.

Due to the linear growth condition on the Lagrangian, the natural energy space to study $(1.1)$ is the space of functions of bounded variation. Recall that if $\Omega$ is an open subset of $\mathbb{R}^{N}$, a function $u \in L^{1}(\Omega)$ whose gradient $D u$ in the sense of distributions is a vector-valued Radon measure with finite total variation in $\Omega$ is called a function of bounded variation. The class of such functions will be denoted by $\operatorname{BV}(\Omega)$. For $u \in$ $\mathrm{BV}(\Omega)$, the vector measure $D u$ decomposes into its absolutely continuous and singular parts, $D u=D^{a} u+D^{s} u$. Then $D^{a} u=\nabla u \mathcal{L}^{N}$, where $\nabla u$ is the Radon-Nikodym derivative of the measure $D u$ with respect to the Lebesgue measure $\mathcal{L}^{N}$. We also split $D^{s} u$ in two parts: the jump part $D^{j} u$ and the Cantor part $D^{c} u$. It is well known (see for instance [1]) that

$$
D^{j} u=\left(u^{+}-u^{-}\right) v_{u} \mathcal{H}^{N-1}\left\llcorner J_{u},\right.
$$

where $J_{u}$ denotes the set of approximate jump points of $u$, and $v_{u}(x)=\frac{D u}{|D u|}(x), D u /|D u|$ being the Radon-Nikodym derivative of $D u$ with respect to its total variation $|D u|$. For further information concerning functions of bounded variation we refer to [1], [23] or [29]. 


\subsection{Lower semicontinuity of functionals defined on BV}

Let $\Omega$ be an open subset of $\mathbb{R}^{N}$. Given a Borel function $g: \Omega \times \mathbb{R} \times \mathbb{R}^{N} \rightarrow[0, \infty)$, we consider the energy functional

$$
G(u):=\int_{\Omega} g(x, u(x), \nabla u(x)) d x
$$

defined in the Sobolev space $W^{1,1}(\Omega)$. In order to get an integral representation of the relaxed energy associated with $G$, i.e.,

$$
\mathcal{G}(u):=\inf _{\left\{u_{n}\right\}}\left\{\liminf _{n \rightarrow \infty} G\left(u_{n}\right): u_{n} \in W^{1,1}(\Omega), u_{n} \rightarrow u \in L^{1}(\Omega)\right\},
$$

Dal Maso introduced in [18] the following functional for $u \in \operatorname{BV}(\Omega)$ :

$$
\begin{aligned}
\mathcal{R}_{g}(u):= & \int_{\Omega} g(x, u(x), \nabla u(x)) d x+\int_{\Omega} g^{0}\left(x, \tilde{u}(x), \frac{D u}{|D u|}(x)\right)\left|D^{c} u\right| \\
& +\int_{J_{u}}\left(\int_{u_{-}(x)}^{u_{+}(x)} g^{0}\left(x, s, v_{u}(x)\right) d s\right) d \mathcal{H}^{N-1}(x),
\end{aligned}
$$

where the recession function $g^{0}$ of $g$ is defined as

$$
g^{0}(x, z, \xi)=\lim _{t \rightarrow 0^{+}} \operatorname{tg}(x, z, \xi / t) .
$$

In the case that $\Omega$ is a bounded set, and under standard continuity and coercivity assumptions, Dal Maso proved in [18] that $\mathcal{G}(u)=\mathcal{R}_{g}(u)$ for all $u \in \mathrm{BV}(\Omega)$. Recently, De Cicco, Fusco, and Verde [20] have obtained a very general result about the $L^{1}$-lower semicontinuity of $\mathcal{R}_{g}$ in $\mathrm{BV}$, which contains, in particular, the following statement.

Theorem 2.1. Let $\Omega$ be an open subset of $\mathbb{R}^{N}$ and $g: \Omega \times \mathbb{R} \times \mathbb{R}^{N} \rightarrow[0, \infty) a$ locally bounded Carathéodory function such that, for every $(z, \xi) \in \mathbb{R} \times \mathbb{R}^{N}$, the function $g(\cdot, z, \xi)$ is of class $C^{1}$. Assume that

(i) $g(x, z, \cdot)$ is convex in $\mathbb{R}^{N}$ for every $(x, z) \in \Omega \times \mathbb{R}$,

(ii) $g(x, \cdot, \xi)$ is continuous in $\mathbb{R}$ for every $(x, \xi) \in \Omega \times \mathbb{R}^{N}$.

Then the functional $\mathcal{R}_{g}(u)$ is lower semicontinuous with respect to the $L^{1}(\Omega)$-convergence.

Let $f: \mathbb{R} \times \mathbb{R}^{N} \rightarrow[0, \infty)$ be a continuous function such that $f^{0}$ exists and $\left|f^{0}(z, \xi)\right| \leq$ $M\|\xi\|$ for any $z \in \mathbb{R}, \xi \in \mathbb{R}^{N}$. Given a function $u \in \mathrm{BV}\left(\mathbb{R}^{N}\right)$, we define the Radon measure $f(u, D u)$ in $\mathbb{R}^{N}$ as

$$
\langle f(u, D u), \phi\rangle:=\mathcal{R}_{\phi f}(u), \quad \phi \in C_{c}\left(\mathbb{R}^{N}\right) .
$$


Observe that if $f^{0}(z, \xi)=\varphi(z) \psi^{0}(\xi)$, where $\varphi$ is Lipschitz continuous and $\psi^{0}$ is a homogeneous function of degree 1 , by applying the chain rule for BV-functions (see [1]), we have

$$
\mathcal{R}_{\phi f}(u)=\int_{\mathbb{R}^{N}} \phi(x) f(u, \nabla u) d x+\int_{\mathbb{R}^{N}} \phi(x) \psi^{0}\left(\frac{D u}{|D u|}\right)\left|D^{s} J_{\varphi}(u)\right|,
$$

where $J_{\varphi}(r)=\int_{0}^{r} \varphi(s) d s$. Then, under these conditions, we have

$$
f(u, D u)^{s}=\psi^{0}\left(\frac{D u}{|D u|}\right)\left|D^{s} J_{\varphi}(u)\right|
$$

\subsection{A generalized Green formula}

We shall need several results from [8] (see also [3]) in order to give a meaning to integrals of bounded vector fields with divergence in $L^{1}$ integrated with respect to the gradient of a BV function. Following [8], we define

$$
X_{1}\left(\mathbb{R}^{N}\right)=\left\{\mathbf{z} \in L^{\infty}\left(\mathbb{R}^{N}, \mathbb{R}^{N}\right): \operatorname{div}(\mathbf{z}) \in L^{1}\left(\mathbb{R}^{N}\right)\right\}
$$

If $\mathbf{z} \in X_{1}\left(\mathbb{R}^{N}\right)$ and $w \in \operatorname{BV}\left(\mathbb{R}^{N}\right) \cap L^{\infty}\left(\mathbb{R}^{N}\right)$ we define the functional (z, Dw) : $C_{c}^{\infty}\left(\mathbb{R}^{N}\right) \rightarrow \mathbb{R}$ by the formula

$$
\langle(\mathbf{z}, D w), \varphi\rangle:=-\int_{\mathbb{R}^{N}} w \varphi \operatorname{div}(\mathbf{z}) d x-\int_{\mathbb{R}^{N}} w \mathbf{z} \cdot \nabla \varphi d x .
$$

Then $(\mathbf{z}, D w)$ is a Radon measure in $\mathbb{R}^{N}$, and

$$
\int_{\mathbb{R}^{N}}(\mathbf{z}, D w)=\int_{\mathbb{R}^{N}} \mathbf{z} \cdot \nabla w d x, \quad \forall w \in W^{1,1}\left(\mathbb{R}^{N}\right) \cap L^{\infty}\left(\mathbb{R}^{N}\right) .
$$

Moreover, $(\mathbf{z}, D w)$ is absolutely continuous with respect to $|D w|$. Its Radon-Nikodym derivative, denoted by $\theta(\mathbf{z}, D w, x)$, is a $|D w|$-measurable function from $\mathbb{R}^{N}$ to $\mathbb{R}$ such that

$$
\int_{B}(\mathbf{z}, D w)=\int_{B} \theta(\mathbf{z}, D w, x)|D w| \quad \text { for any Borel set } B \subseteq \mathbb{R}^{N} .
$$

By writing

$$
\mathbf{z} \cdot D^{s} u:=(\mathbf{z}, D u)-(\mathbf{z} \cdot \nabla u) d \mathcal{L}^{N}
$$

we see that $\mathbf{z} \cdot D^{s} u$ is a bounded measure.

We have the following Green formula for $\mathbf{z} \in X_{1}\left(\mathbb{R}^{N}\right)$ and $w \in \operatorname{BV}\left(\mathbb{R}^{N}\right) \cap L^{\infty}\left(\mathbb{R}^{N}\right)$ ([8]):

$$
\int_{\mathbb{R}^{N}} w \operatorname{div}(\mathbf{z}) d x+\int_{\mathbb{R}^{N}}(\mathbf{z}, D w)=0 .
$$




\section{The elliptic problem}

\subsection{Assumptions on the Lagrangian $f$}

Our purpose in this section is to introduce the main assumptions on the Lagrangian $f$ and recall the meaning of the expression

$$
v=-\operatorname{div} \mathbf{a}(u, D u) \quad \text { in } \mathbb{R}^{N}
$$

according to [6].

We assume that the Lagrangian $f: \mathbb{R} \times \mathbb{R}^{N} \rightarrow \mathbb{R}^{+}$satisfies the following assumptions, to which we refer collectively as $(\mathrm{H})$ :

$\left(\mathrm{H}_{1}\right) f$ is continuous on $\mathbb{R} \times \mathbb{R}^{N}$ and is a convex differentiable function of $\xi$ such that $\nabla_{\xi} f(z, \xi) \in C\left(\mathbb{R} \times \mathbb{R}^{N}\right)$. Further we require $f$ to satisfy the linear growth condition

$$
C_{0}(z)\|\xi\|-D_{0}(z) \leq f(z, \xi) \leq M_{0}(z)(\|\xi\|+1)
$$

for any $(z, \xi) \in \mathbb{R} \times \mathbb{R}^{N}$, and some positive and continuous functions $C_{0}, D_{0}, M_{0}$, such that $C_{0}(z)>0$ for any $z \neq 0$. Moreover, we assume $f^{0}$ exists.

We consider the function $\mathbf{a}(z, \xi)=\nabla_{\xi} f(z, \xi)$ associated to the Lagrangian $f$. By the convexity of $f$,

$$
\mathbf{a}(z, \xi) \cdot(\eta-\xi) \leq f(z, \eta)-f(z, \xi),
$$

and the following monotonicity condition is satisfied:

$$
(\mathbf{a}(z, \eta)-\mathbf{a}(z, \xi)) \cdot(\eta-\xi) \geq 0 .
$$

Moreover, it is easy to see that for each $R>0$, there is a constant $M=M(R)>0$ such that

$$
|\mathbf{a}(z, \xi)| \leq M \quad \forall(z, \xi) \in \mathbb{R} \times \mathbb{R}^{N},|z| \leq R .
$$

We also assume that $\mathbf{a}(z, 0)=0$ for all $z \in \mathbb{R}$, and $\mathbf{a}(z, \xi)=z \mathbf{b}(z, \xi)$ with

$$
|\mathbf{b}(z, \xi)| \leq M_{0} \quad \forall(z, \xi) \in \mathbb{R} \times \mathbb{R}^{N},|z| \leq R .
$$

We consider the function $h: \mathbb{R} \times \mathbb{R}^{N} \rightarrow \mathbb{R}$ defined by

$$
h(z, \xi):=\mathbf{a}(z, \xi) \cdot \xi .
$$

By (3.4), we have

$$
h(z, \xi) \geq 0 \quad \forall \xi \in \mathbb{R}^{N}, z \in \mathbb{R} .
$$

Moreover, from (3.3) and (3.2), it follows that

$$
C_{0}(z)\|\xi\|-D_{1}(z) \leq h(z, \xi) \leq M\|\xi\|
$$


for any $(z, \xi) \in \mathbb{R} \times \mathbb{R}^{N},|z| \leq R$, where $D_{1}(z)=D_{0}(z)+f(z, 0)$. We note that the left inequality holds for any $(z, \xi) \in \mathbb{R} \times \mathbb{R}^{N}$. Moreover, we assume that there exist constants $A, B>0$ and $\alpha, \beta \geq 1$ such that

$$
\left|D_{1}(z)\right| \leq A|z|^{\alpha}+B|z|^{\beta} \quad \text { for any } z \in \mathbb{R}^{N} .
$$

This condition will be used to prove some estimates during the proof of existence, and we assume it for simplicity, since a more general condition could be used.

We assume that

$\left(\mathrm{H}_{2}\right) \frac{\partial \mathbf{a}}{\partial \xi_{i}}(z, \xi) \in C\left(\mathbb{R} \times \mathbb{R}^{N}\right)$ for any $i=1, \ldots, N$.

$\left(\mathrm{H}_{3}\right) h(z, \xi)=h(z,-\xi)$ for all $z \in \mathbb{R}$ and $\xi \in \mathbb{R}^{N}$, and $h^{0}$ exists.

Observe that

$$
C_{0}(z)\|\xi\| \leq h^{0}(z, \xi) \leq M\|\xi\| \quad \text { for any }(z, \xi) \in \mathbb{R} \times \mathbb{R}^{N},|z| \leq R .
$$

$\left(\mathrm{H}_{4}\right) f^{0}(z, \xi)=h^{0}(z, \xi)$ for all $\xi \in \mathbb{R}^{N}$ and all $z \in \mathbb{R}$.

( $\left.\mathrm{H}_{5}\right) \mathbf{a}(z, \xi) \cdot \eta \leq h^{0}(z, \eta)$ for all $\xi, \eta \in \mathbb{R}^{N}$ and all $z \in \mathbb{R}$.

$\left(\mathrm{H}_{6}\right) h^{0}(z, \xi)$ can be written in the form $h^{0}(z, \xi)=\varphi(z) \psi^{0}(\xi)$, where $\varphi$ is a Lipschitz continuous function such that $\varphi(z)>0$ for any $z \neq 0$, and $\psi^{0}$ is a convex function which is homogeneous of degree 1 .

$\left(\mathrm{H}_{7}\right)$ For any $R>0$, there is a constant $C>0$ such that

$$
|(\mathbf{a}(z, \xi)-\mathbf{a}(\hat{z}, \xi)) \cdot(\xi-\hat{\xi})| \leq C|z-\hat{z}|\|\xi-\hat{\xi}\|
$$

for any $(z, \xi),(\hat{z}, \hat{\xi}) \in \mathbb{R} \times \mathbb{R}^{N},|z|,|\hat{z}| \leq R$.

Observe that, by the monotonicity condition 3.4 and using 3.10, it follows that

$$
(\mathbf{a}(z, \xi)-\mathbf{a}(\hat{z}, \hat{\xi})) \cdot(\xi-\hat{\xi}) \geq-C|z-\hat{z}|\|\xi-\hat{\xi}\|
$$

for any $(z, \xi),(\hat{z}, \xi) \in \mathbb{R} \times \mathbb{R}^{N},|z|,|\hat{z}| \leq R$.

Observe that under assumptions $\left(\mathrm{H}_{4}\right)$ and $\left(\mathrm{H}_{6}\right)$, applying 2.5], we have

$$
f(u, D u)^{s}=h(u, D u)^{s}=\psi^{0}\left(\frac{D u}{|D u|}\right)\left|D^{s} J_{\varphi}(u)\right|
$$

Remark 3.1. There are physical models for plasma fusion by inertial confinement in which the temperature evolution of the electrons satisfies an equation of type (1.1), where

$$
\mathbf{a}(z, \xi)=\frac{|z|^{5 / 2} \xi}{1+|z||\xi|},
$$

which corresponds to $f(z, \xi)=|z|^{3 / 2}|\xi|-|z|^{1 / 2} \ln (1+|z||\xi|)$ [22] (see also [13] for a mathematical study in the one-dimensional case). It is easy to check that $\left(\mathrm{H}_{1}\right)$ (in particular 3.2 and $(3.8)$ ) holds for any $(z, \xi) \in \mathbb{R} \times \mathbb{R}^{N}$. Notice that condition $\left(\mathrm{H}_{2}\right)$ holds. We 
observe that $h^{0}(z, \xi)=|z|^{3 / 2}|\xi|$ and $\left(\mathrm{H}_{3}\right)-\left(\mathrm{H}_{6}\right)$ hold. Finally, to check $\left(\mathrm{H}_{7}\right)$ we observe that

$$
\frac{\partial \mathbf{a}}{\partial z}(z, \xi)=\frac{5}{2} \frac{z^{3 / 2} \xi}{1+z|\xi|}-\frac{z^{5 / 2}|\xi| \xi}{(1+z|\xi|)^{2}}
$$

and therefore

$$
\left|\frac{\partial \mathbf{a}}{\partial z}(z, \xi)\right| \leq \frac{7}{2}|z|^{1 / 2}
$$

for any $(z, \xi) \in \mathbb{R} \times \mathbb{R}^{N}$. From this, it follows that

$$
|\mathbf{a}(z, \xi)-\mathbf{a}(\hat{z}, \xi)| \leq \frac{7}{2} R^{1 / 2}|z-\hat{z}|
$$

for any $(z, \xi) \in \mathbb{R} \times \mathbb{R}^{N},|z| \leq R$. Thus also $\left(\mathrm{H}_{7}\right)$ holds. In this case, the results below will prove existence and uniqueness of entropy solutions of 1.1 for any initial condition $u_{0} \in L^{\infty}\left(\mathbb{R}^{N}\right) \cap L^{1}\left(\mathbb{R}^{N}\right), u_{0} \geq 0$.

Remark 3.2. The function $f(z, \xi)=\left(v / a^{2}\right)|z| \sqrt{z^{2}+a^{2}|\xi|^{2}}$ satisfies the assumptions $\left(\mathrm{H}_{1}\right)-\left(\mathrm{H}_{7}\right)$, with

$$
\mathbf{a}(z, \xi)=v \frac{|z| \xi}{\sqrt{z^{2}+a^{2}|\xi|^{2}}} .
$$

This particular case is related to the so-called relativistic heat equation (see [14], [27])

$$
u_{t}=v \operatorname{div}\left(\frac{|u| D u}{\sqrt{u^{2}+a^{2}|D u|^{2}}}\right)
$$

with $a=v / c, c$ being a bound of the propagation speed, and $v$ being a constant representing a kinematic viscosity.

Let us mention that, as pointed out by Brenier in [14], this relativistic heat equation can be derived using Monge-Kantorovich's mass transport theory. On the other hand, it is among the various flux limited diffusion equations used in the theory of radiation hydrodynamics [25]. Indeed, a very similar equation

$$
u_{t}=v \operatorname{div}\left(\frac{u D u}{u+\frac{v}{c}|D u|}\right)
$$

can be found in [25]. In this case, the Lagrangian $f$ associated with the above equation is

$$
f(z, \xi):=c z\left(|\xi|-\frac{c z}{v} \log \left(1+\frac{v}{c z}|\xi|\right)\right),
$$

and satisfies assumptions $\left(\mathrm{H}_{1}\right)-\left(\mathrm{H}_{7}\right)$. 


\subsection{A functional calculus}

We need to consider the following truncature functions. For $a<b$, let $T_{a, b}(r):=$ $\max (\min (b, r), a)$. As usual, we write $T_{k}=T_{-k, k}$. We also consider the truncature functions $T_{a, b}^{l}(r):=T_{a, b}(r)-l(l \in \mathbb{R})$. We set

$$
\mathcal{T}_{r}:=\left\{T_{a, b}: 0<a<b\right\}, \quad \mathcal{T}^{+}:=\left\{T_{a, b}^{l}: 0<a<b, l \in \mathbb{R}, T_{a, b}^{l} \geq 0\right\} .
$$

We need to consider the function space

$$
\operatorname{TBV}^{+}\left(\mathbb{R}^{N}\right):=\left\{u \in L^{1}\left(\mathbb{R}^{N}\right)^{+}: T(u) \in \mathrm{BV}\left(\mathbb{R}^{N}\right), \forall T \in \mathcal{T}_{r}\right\},
$$

and to give a meaning to the Radon-Nikodym derivative $\nabla u$ of a function $u \in \mathrm{TBV}^{+}\left(\mathbb{R}^{N}\right)$. Using the chain rule for BV functions (see, for instance, [1]), and with a similar proof to the one given in Lemma 2.1 of [9], we obtain the following result.

Lemma 3.3. For every $u \in \mathrm{TBV}^{+}\left(\mathbb{R}^{N}\right)$ there exists a unique measurable function $v$ : $\mathbb{R}^{N} \rightarrow \mathbb{R}^{N}$ such that

$$
\nabla T_{a, b}(u)=v \chi_{[a<u<b]} \quad \mathcal{L}^{N} \text {-a.e., } \forall T_{a, b} \in \mathcal{T}_{r} .
$$

Thanks to this result we define $\nabla u$ for a function $u \in \mathrm{TBV}^{+}\left(\mathbb{R}^{N}\right)$ as the unique function $v$ which satisfies 3.15 . This notation will be used throughout.

We denote by $\mathcal{P}$ the set of Lipschitz continuous function $p:[0, \infty) \rightarrow \mathbb{R}$ satisfying $p^{\prime}(s)=0$ for $s$ large enough. We write $\mathcal{P}^{+}:=\{p \in \mathcal{P}: p \geq 0\}$. We recall the following result ([2, Lemma 2]).

Lemma 3.4. If $u \in \mathrm{TBV}^{+}\left(\mathbb{R}^{N}\right)$, then $p(u) \in \mathrm{BV}\left(\mathbb{R}^{N}\right)$ for every $p \in \mathcal{P}$ such that there exists $a>0$ with $p(r)=0$ for all $0 \leq r \leq a$. Moreover, $\nabla p(u)=p^{\prime}(u) \nabla u \mathcal{L}^{N}$-a.e.

For any function $q$, let $J_{q}(r)$ denote the primitive of $q$, i.e., $J_{q}(r)=\int_{0}^{r} q(s) d s$. Let $S \in \mathcal{P}$ and $T=T_{a, b}^{a}$. Given a function $u \in \mathrm{TBV}^{+}\left(\mathbb{R}^{N}\right)$, by Lemma 3.4 , we have $S(u) T(u), J_{T^{\prime} S}(u), J_{T S^{\prime}}(u) \in \mathrm{BV}\left(\mathbb{R}^{N}\right)$. Moreover, it is easy to see that

$$
D(S(u) T(u))=D J_{T^{\prime} S}(u)+D J_{T S^{\prime}}(u) .
$$

Hence, if $\mathbf{z} \in X_{1}\left(\mathbb{R}^{N}\right)$, we have

$$
(\mathbf{z}, D(S(u) T(u)))=\left(\mathbf{z}, D J_{T^{\prime} S}(u)\right)+\left(\mathbf{z}, D J_{T S^{\prime}}(u)\right) .
$$

Let $g: \mathbb{R}^{N} \times \mathbb{R} \times \mathbb{R}^{N} \rightarrow[0, \infty)$ be a function satisfying the assumption of Theorem 2.1. and $T \in \mathcal{T}^{+}$. Then there is some $T_{a, b} \in \mathcal{T}_{r}$ and a constant $c \in \mathbb{R}$ such that $T=$ $T_{a, b}-c$. Observe that

$$
r=T(r)+c \quad \text { whenever } r \in \mathbb{R} \text { and } T^{\prime}(r)=1 .
$$

Consider the functional

$$
R(g, T)(u):=\int_{\mathbb{R}^{N}} g(x, u(x), \nabla T(u(x))) d x, \quad u \in W^{1,1}\left(\mathbb{R}^{N}\right) .
$$


For $u \in \mathrm{TBV}^{+}\left(\mathbb{R}^{N}\right)$, define

$$
\begin{aligned}
\mathcal{R}(g, T)(u):= & \mathcal{R}_{g}\left(T_{a, b}(u)\right)+\int_{[u \leq a]}(g(x, u(x), 0)-g(x, a, 0)) d x \\
& +\int_{[u \geq b]}(g(x, u(x), 0)-g(x, b, 0)) d x .
\end{aligned}
$$

By Theorem 2.1. $\mathcal{R}(g, T)$ is lower semicontinuous in $\mathrm{TBV}^{+}\left(\mathbb{R}^{N}\right)$ with respect to $L^{1}\left(\mathbb{R}^{N}\right)$-convergence. Observe that, with this notation, we have

$$
\mathcal{R}(g, T)(u)=\mathcal{R}\left(g, T_{a, b}\right)(u) .
$$

Moreover, if $u \in W^{1,1}\left(\mathbb{R}^{N}\right)$, using 3.18) we have

$$
R(g, T)(u)=\mathcal{R}(g, T)(u) .
$$

It will be sufficient for our purposes to assume that $g$ does not depend on $x$. If $u \in$ $\mathrm{TBV}^{+}\left(\mathbb{R}^{N}\right)$ and $T \in \mathcal{T}^{+}$, we define the Radon measure $g(u, D T(u))$ in $\mathbb{R}^{N}$ by

$$
\langle g(u, D T(u)), \phi\rangle:=\mathcal{R}(\phi g, T)(u), \quad \phi \in C_{c}\left(\mathbb{R}^{N}\right) .
$$

If $T \in \mathcal{T}_{r}$, then $T(r)=r$ for any $r \in \mathbb{R}$ such that $T^{\prime}(r)=1$, and, using 3.19, 3.20, and 2.3 , we have

$$
\begin{aligned}
\langle g(u, D T(u)), \phi\rangle= & \langle g(T(u), D T(u)), \phi\rangle+\int_{[u \leq a]} \phi(g(x, u(x), 0)-g(x, a, 0)) d x \\
& +\int_{[u \geq b]} \phi(g(x, u(x), 0)-g(x, b, 0)) d x .
\end{aligned}
$$

Let $S \in \mathcal{P}^{+}$and $T \in \mathcal{T}^{+}$. We denote by $f_{S}(u, D T(u))$ and $h_{S}(u, D T(u))$ the Radon measures defined by $(3.20$ with $g(z, \xi)=S(z) f(z, \xi)$ and $g(z, \xi)=S(z) h(z, \xi)$, respectively. Since $h(z, 0)=0$ for all $z \in \mathbb{R}$, if $S, T \in \mathcal{T}^{+}$, with $T=T_{a, b}-c$, we have

$$
h_{S}(u, D T(u))=h_{S}\left(T_{a, b}(u), D T(u)\right)=h_{S}\left(T_{a, b}(u), D T_{a, b}(u)\right) .
$$

and, by 2.5 ,

$$
\begin{aligned}
\left(f_{S}(u, D T(u))\right)^{s} & =\left(f_{S}\left(T_{a, b}(u), D T_{a, b}(u)\right)\right)^{s} \\
& =\psi^{0}\left(\frac{D T_{a, b}(u)}{\left|D T_{a, b}(u)\right|}\right)\left|D^{s} J_{S \varphi}\left(T_{a, b}(u)\right)\right| .
\end{aligned}
$$

Similarly, we have

$$
\left(h_{S}(u, D T(u))\right)^{s}=\left(h_{S}\left(u, D T_{a, b}(u)\right)\right)^{s}=\psi^{0}\left(\frac{D T_{a, b}(u)}{\left|D T_{a, b}(u)\right|}\right)\left|D^{s} J_{S \varphi}\left(T_{a, b}(u)\right)\right| .
$$

Note that both singular parts are identical. By the representation formulas in Subsection 2.2. the absolutely continuous part of $h_{S}(u, D T(u))$ is $S(u) h(u, \nabla T(u))$. Similar identities are true when $S=1$. 


\subsection{An existence and uniqueness result for the elliptic problem}

Let us recall the following concept of solution for problem 3.1 introduced in [6].

Definition 3.5. Given $v \in L^{\infty}\left(\mathbb{R}^{N}\right) \cap L^{1}\left(\mathbb{R}^{N}\right), v \geq 0$, we say that $u \geq 0$ is an entropy solution of $\sqrt{3.1}$ if $u \in \mathrm{TBV}^{+}\left(\mathbb{R}^{N}\right)$ and $\mathbf{a}(u, \nabla u) \in X_{1}\left(\mathbb{R}^{N}\right)$ satisfies

$$
\begin{aligned}
& v=-\operatorname{div} \mathbf{a}(u, \nabla u)) \quad \text { in } \mathcal{D}^{\prime}\left(\mathbb{R}^{N}\right), \\
& h_{S}(u, D T(u)) \leq\left(\mathbf{a}(u, \nabla u), D J_{T^{\prime} S}(u)\right) \quad \text { as measures } \forall S \in \mathcal{P}^{+}, T \in \mathcal{T}^{+} \text {, } \\
& h(u, D T(u)) \leq(\mathbf{a}(u, \nabla u), D T(u)) \quad \text { as measures } \forall T \in \mathcal{T}^{+} \text {. }
\end{aligned}
$$

Note that 3.25 and 3.26 are equivalent to

$$
h_{S}(u, D T(u))^{S} \leq\left(\mathbf{a}(u, \nabla u), D J_{T^{\prime} S}(u)\right)^{s} \quad \text { as measures } \forall S \in \mathcal{P}^{+}, T \in \mathcal{T}^{+} \text {, }
$$

and

$$
h(u, D T(u))^{s} \leq(\mathbf{a}(u, \nabla u), D T(u))^{s} \quad \text { as measures } \forall T \in \mathcal{T}^{+},
$$

respectively.

The main result of [6] is the following existence and uniqueness result.

Theorem 3.6. Assume that assumptions $(\mathrm{H})$ hold. Then for any $0 \leq v \in L^{\infty}\left(\mathbb{R}^{N}\right) \cap$ $L^{1}\left(\mathbb{R}^{N}\right)$ there exists a unique entropy solution $u \in \mathrm{TBV}^{+}\left(\mathbb{R}^{N}\right) \cap L^{\infty}\left(\mathbb{R}^{N}\right)$ of the problem

$$
u-\operatorname{div} \mathbf{a}(u, D u)=v \quad \text { in } \mathbb{R}^{N} .
$$

Moreover, given $v, \bar{v} \in L^{\infty}\left(\mathbb{R}^{N}\right)^{+}$, if $u, \bar{u}$ are bounded entropy solutions of the problems

$$
u-\operatorname{div} \mathbf{a}(u, D u)=v \quad \text { in } \mathbb{R}^{N}
$$

and

$$
\bar{u}-\operatorname{div} \mathbf{a}(\bar{u}, D \bar{u})=\bar{v} \quad \text { in } \mathbb{R}^{N},
$$

respectively, then

$$
\int_{\mathbb{R}^{N}}(u-\bar{u})^{+} \leq \int_{\mathbb{R}^{N}}(v-\bar{v})^{+}
$$

\subsection{Semigroup solution}

Following [6], we associate to the formal differential expression $-\operatorname{div} \mathbf{a}(u, \nabla u)$ the following operator in $L^{1}\left(\mathbb{R}^{N}\right)$.

Definition 3.7. $(u, v) \in B$ if and only if $0 \leq u \in \mathrm{TBV}^{+}\left(\mathbb{R}^{N}\right) \cap L^{\infty}\left(\mathbb{R}^{N}\right), 0 \leq v \in$ $L^{\infty}\left(\mathbb{R}^{N}\right) \cap L^{1}\left(\mathbb{R}^{N}\right)$ and $u$ is the entropy solution of problem 3.1 .

The following result was proved in [6]. 
Proposition 3.8. Under assumptions $(\mathrm{H})$, the operator $B$ is accretive in $L^{1}\left(\mathbb{R}^{N}\right)$,

$$
\left(L^{\infty}\left(\mathbb{R}^{N}\right) \cap L^{1}\left(\mathbb{R}^{N}\right)\right)^{+} \subset R(I+B)
$$

and $D(B)$ is dense in $L^{1}\left(\mathbb{R}^{N}\right)^{+}$. Moreover, given $\lambda>0$ and $v \in L^{1}\left(\mathbb{R}^{N}\right)^{+}$,

$$
u=(I+\lambda B)^{-1} v<<v .
$$

From Proposition 3.8 , if we denote by $\mathcal{B}$ the closure in $L^{1}\left(\mathbb{R}^{N}\right)$ of the operator $B$, it follows that $\mathcal{B}$ is accretive in $L^{1}\left(\mathbb{R}^{N}\right)$, and satisfies the comparison principle and the range condition $\overline{D(\mathcal{B})}{ }^{L^{1}\left(\mathbb{R}^{N}\right)}=L^{1}\left(\mathbb{R}^{N}\right)^{+} \subset R(I+\lambda \mathcal{B})$ for all $\lambda>0$. Therefore, according to Crandall-Liggett's Theorem (cf., e.g., [11]), for any $0 \leq u_{0} \in L^{1}\left(\mathbb{R}^{N}\right)$ there exists a unique mild solution $u \in C\left([0, T], L^{1}\left(\mathbb{R}^{N}\right)\right)$ of the abstract Cauchy problem

$$
u^{\prime}(t)+\mathcal{B} u(t) \ni 0, \quad u(0)=u_{0} .
$$

Moreover, $u(t)=T(t) u_{0}$ for all $t \geq 0$, where $(T(t))_{t \geq 0}$ is the semigroup in $L^{1}\left(\mathbb{R}^{N}\right)^{+}$ generated by Crandall-Liggett's exponential formula, i.e.,

$$
T(t) u_{0}=\lim _{n \rightarrow \infty}\left(I+\frac{t}{n} \mathcal{B}\right)^{-n} u_{0} .
$$

On the other hand, by [3.30], and the results in [10], the comparison principle also holds for $T(t)$, i.e., if $u_{0}, \bar{u}_{0} \in L^{1}\left(\mathbb{R}^{N}\right)^{+}$, we have the estimate

$$
\left\|\left(T(t) u_{0}-T(t) \bar{u}_{0}\right)^{+}\right\|_{1} \leq\left\|\left(u_{0}-\bar{u}_{0}\right)^{+}\right\|_{1} .
$$

Remark 3.9. Since, by Proposition 3.8, $\left(L^{\infty}\left(\mathbb{R}^{N}\right) \cap L^{1}\left(\mathbb{R}^{N}\right)\right)^{+} \subset R(I+B)$, using 3.30, we have

$$
T(t) u_{0} \in\left(L^{\infty}\left(\mathbb{R}^{N}\right) \cap L^{1}\left(\mathbb{R}^{N}\right)\right)^{+} \quad \forall t \geq 0, \forall u_{0} \in\left(L^{\infty}\left(\mathbb{R}^{N}\right) \cap L^{1}\left(\mathbb{R}^{N}\right)\right)^{+} .
$$

Remark 3.10. In the proof of the existence part of Theorem 3.6 (see [6]), we have proved that the resolvent of the operator $B_{n}$ associated to $-\operatorname{div} \mathbf{a}(u, D u)-\frac{1}{n} \Delta u$ converges to the resolvent of $B$, i.e., if $v \in L^{1}\left(\mathbb{R}^{N}\right) \cap L^{\infty}\left(\mathbb{R}^{N}\right), v \geq 0$, and $u_{n}$ are solutions of $\left(I+B_{n}\right) u=v$, then $u_{n} \rightarrow u$ in $L^{1}\left(\mathbb{R}^{N}\right)$ (and in $L^{p}\left(\mathbb{R}^{N}\right)$ for all $\left.1 \leq p<\infty\right)$ where $u=(I+B)^{-1} v$.

\section{Existence and uniqueness of solutions of the parabolic problem}

In this section we give the concept of entropy solution for the Cauchy problem (1.1) and we state the existence and uniqueness result for this type of solution.

To make precise our notion of solution we need to recall several definitions given in [2].

We define the space

$$
Z\left(\mathbb{R}^{N}\right):=\left\{(\mathbf{z}, \xi) \in L^{\infty}\left(\mathbb{R}^{N}, \mathbb{R}^{N}\right) \times \mathrm{BV}\left(\mathbb{R}^{N}\right)^{*}: \operatorname{div}(\mathbf{z})=\xi \text { in } \mathcal{D}^{\prime}\left(\mathbb{R}^{N}\right)\right\} .
$$


We need to consider the space $\mathrm{BV}\left(\mathbb{R}^{N}\right)_{2}$, defined as $\mathrm{BV}\left(\mathbb{R}^{N}\right) \cap L^{2}\left(\mathbb{R}^{N}\right)$ endowed with the norm

$$
\|w\|_{\mathrm{BV}\left(\mathbb{R}^{N}\right)_{2}}:=\|w\|_{L^{2}\left(\mathbb{R}^{N}\right)}+|D w|\left(\mathbb{R}^{N}\right) .
$$

It is easy to see that $L^{2}\left(\mathbb{R}^{N}\right) \subset \mathrm{BV}\left(\mathbb{R}^{N}\right)_{2}^{*}$ and

$$
\|w\|_{\mathrm{BV}\left(\mathbb{R}^{N}\right)_{2}^{*}} \leq\|w\|_{L^{2}\left(\mathbb{R}^{N}\right)} \quad \forall w \in L^{2}\left(\mathbb{R}^{N}\right) .
$$

It is well known (see [28]) that the dual space $\left(L^{1}\left(0, T, \mathrm{BV}\left(\mathbb{R}^{N}\right)_{2}\right)\right)^{*}$ is isometric to the space $L^{\infty}\left(0, T ; \mathrm{BV}\left(\mathbb{R}^{N}\right)_{2}^{*}, \mathrm{BV}\left(\mathbb{R}^{N}\right)_{2}\right)$ of all weak* measurable functions $f$ : $[0, T] \rightarrow \mathrm{BV}\left(\mathbb{R}^{N}\right)_{2}^{*}$ such that $v(f) \in L^{\infty}([0, T])$, where $v(f)$ denotes the supremum of the set $\left\{|\langle w, f\rangle|:\|w\|_{\mathrm{BV}\left(\mathbb{R}^{N}\right)_{2}} \leq 1\right\}$ in the vector lattice of measurable real functions. Moreover, the duality pairing is

$$
\langle w, f\rangle=\int_{0}^{T}\langle w(t), f(t)\rangle d t
$$

for $w \in L^{1}\left(0, T, \mathrm{BV}\left(\mathbb{R}^{N}\right)_{2}\right)$ and $f \in L^{\infty}\left(0, T ; \mathrm{BV}\left(\mathbb{R}^{N}\right)_{2}^{*}, \mathrm{BV}\left(\mathbb{R}^{N}\right)_{2}\right)$.

We denote by $L_{w}^{1}\left(0, T, \mathrm{BV}\left(\mathbb{R}^{N}\right)\right)$ the space of weakly measurable functions $w$ : $[0, T] \rightarrow \mathrm{BV}\left(\mathbb{R}^{N}\right)$ (i.e., $t \in[0, T] \mapsto\langle w(t), \phi\rangle$ is measurable for every $\phi \in \mathrm{BV}\left(\mathbb{R}^{N}\right)^{*}$ ) such that $\int_{0}^{T}\|w(t)\| d t<\infty$. Observe that, since $\mathrm{BV}\left(\mathbb{R}^{N}\right)$ has a separable predual (see [1]), it follows easily that the map $t \in[0, T] \mapsto\|w(t)\|$ is measurable. We denote by $L_{\text {loc }, w}^{1}\left(0, T, \mathrm{BV}\left(\mathbb{R}^{N}\right)\right)$ the space of weakly measurable functions $w:[0, T] \rightarrow \mathrm{BV}\left(\mathbb{R}^{N}\right)$ such that the map $t \in[0, T] \mapsto\|w(t)\|$ is in $L_{\text {loc }}^{1}((0, T))$.

Let us recall the following definitions given in [2].

Definition 4.1. Let $\Psi \in L^{1}\left(0, T, \mathrm{BV}\left(\mathbb{R}^{N}\right)\right)$. We say $\Psi$ admits $a$ weak derivative in the space $L_{w}^{1}\left(0, T, \mathrm{BV}\left(\mathbb{R}^{N}\right)\right) \cap L^{\infty}\left(Q_{T}\right)$ if there is $\Theta \in L_{w}^{1}\left(0, T, \mathrm{BV}\left(\mathbb{R}^{N}\right)\right) \cap L^{\infty}\left(Q_{T}\right)$ such that $\Psi(t)=\int_{0}^{t} \Theta(s) d s$, the integral being a Pettis integral ([21]).

Definition 4.2. Let $\xi \in\left(L^{1}\left(0, T, \mathrm{BV}\left(\mathbb{R}^{N}\right)_{2}\right)\right)^{*}$. We say that $\xi$ is the time derivative in the space $\left(L^{1}\left(0, T, \mathrm{BV}\left(\mathbb{R}^{N}\right)_{2}\right)\right)^{*}$ of a function $u \in L^{1}\left((0, T) \times \mathbb{R}^{N}\right)$ if

$$
\int_{0}^{T}\langle\xi(t), \Psi(t)\rangle d t=-\int_{0}^{T} \int_{\mathbb{R}^{N}} u(t, x) \Theta(t, x) d x d t
$$

for all test functions $\Psi \in L^{1}\left(0, T, \mathrm{BV}\left(\mathbb{R}^{N}\right)\right)$ with compact support in time, which admit a weak derivative $\Theta \in L_{w}^{1}\left(0, T, \mathrm{BV}\left(\mathbb{R}^{N}\right)\right) \cap L^{\infty}\left(Q_{T}\right)$.

Note that if $w \in L^{1}\left(0, T, \operatorname{BV}\left(\mathbb{R}^{N}\right)\right) \cap L^{\infty}\left(Q_{T}\right)$ and $\mathbf{z} \in L^{\infty}\left(Q_{T}, \mathbb{R}^{N}\right)$ is such that there exists $\xi \in\left(L^{1}\left(0, T, \operatorname{BV}\left(\mathbb{R}^{N}\right)\right)\right)^{*}$ with $\operatorname{div}(\mathbf{z})=\xi$ in $\mathcal{D}^{\prime}\left(Q_{T}\right)$, we can define, associated to the pair $(\mathbf{z}, \xi)$, the distribution $(\mathbf{z}, D w)$ in $Q_{T}$ by

$$
\langle(\mathbf{z}, D w), \phi\rangle:=-\int_{0}^{T}\langle\xi(t), w(t) \phi(t)\rangle d t-\int_{0}^{T} \int_{\mathbb{R}^{N}} \mathbf{z}(t, x) w(t, x) \nabla_{x} \phi(t, x) d x d t
$$

for all $\phi \in \mathcal{D}\left(Q_{T}\right)$. 
Definition 4.3. Let $\xi \in\left(L^{1}\left(0, T, \mathrm{BV}\left(\mathbb{R}^{N}\right)_{2}\right)\right)^{*}$ and $\mathbf{z} \in L^{\infty}\left(Q_{T}, \mathbb{R}^{N}\right)$. We say that $\xi=$ $\operatorname{div}(\mathbf{z})$ in $\left(L^{1}\left(0, T, \mathrm{BV}\left(\mathbb{R}^{N}\right)_{2}\right)\right)^{*}$ if $(\mathbf{z}, D w)$ is a Radon measure in $Q_{T}$ such that

$$
\int_{Q_{T}}(\mathbf{z}, D w)+\int_{0}^{T}\langle\xi(t), w(t)\rangle d t=0
$$

for all $w \in L^{1}\left(0, T, \mathrm{BV}\left(\mathbb{R}^{N}\right)\right) \cap L^{\infty}\left(Q_{T}\right)$.

Our concept of solution for problem (1.1) is the following.

Definition 4.4. A measurable function $u:(0, T) \times \mathbb{R}^{N} \rightarrow \mathbb{R}$ is an entropy solution of 1.1 in $Q_{T}=(0, T) \times \mathbb{R}^{N}$ if $u \in C\left([0, T], L^{1}\left(\mathbb{R}^{N}\right)\right), T_{a, b}(u(\cdot)) \in L_{\mathrm{loc}, w}^{1}\left(0, T, \mathrm{BV}\left(\mathbb{R}^{N}\right)\right)$ for all $0<a<b$, and there exists $\xi \in\left(L^{1}\left(0, T, \mathrm{BV}\left(\mathbb{R}^{N}\right)_{2}\right)^{*}\right.$ such that:

(i) $(\mathbf{a}(u(t), \nabla u(t)), \xi(t)) \in Z\left(\mathbb{R}^{N}\right)$ a.e. in $t \in[0, T]$,

(ii) $\xi$ is the time derivative of $u$ in $\left(L^{1}\left(0, T, \mathrm{BV}\left(\mathbb{R}^{N}\right)_{2}\right)\right)^{*}$ in the sense of Definition 4.2

(iii) $\xi=\operatorname{div} \mathbf{a}(u(t), \nabla u(t))$ in the sense of Definition 4.3. and

(iv) the following inequality is satisfied:

$$
\begin{aligned}
& \int_{0}^{T} \int_{\mathbb{R}^{N}} \phi h_{S}(u, D T(u)) d t+\int_{0}^{T} \int_{\mathbb{R}^{N}} \phi h_{T}(u, D S(u)) d t \\
& \leq \int_{0}^{T} \int_{\mathbb{R}^{N}} J_{T S}(u(t)) \phi^{\prime}(t) d x d t-\int_{0}^{T} \int_{\mathbb{R}^{N}} \mathbf{a}(u(t), \nabla u(t)) \cdot \nabla \phi T(u(t)) S(u(t)) d x d t
\end{aligned}
$$

for truncatures $S, T \in \mathcal{T}^{+}$and any smooth function $\phi$ of compact support, in particular of the form $\phi(t, x)=\phi_{1}(t) \rho(x), \phi_{1} \in \mathcal{D}((0, T)), \rho \in \mathcal{D}\left(\mathbb{R}^{N}\right), \phi_{1}, \rho \geq 0$.

We have the following existence and uniqueness result.

Theorem 4.5. Under assumptions $(\mathrm{H})$, for any initial datum $0 \leq u_{0} \in L^{\infty}\left(\mathbb{R}^{N}\right) \cap$ $L^{1}\left(\mathbb{R}^{N}\right)$ there exists a unique entropy solution $u$ of 1.1 in $Q_{T}=(0, T) \times \mathbb{R}^{N}$ for every $T>0$ such that $u(0)=u_{0}$. Moreover, if $u(t), \bar{u}(t)$ are the entropy solutions corresponding to initial data $u_{0}, \bar{u}_{0} \in\left(L^{\infty}\left(\mathbb{R}^{N}\right) \cap L^{1}\left(\mathbb{R}^{N}\right)\right)^{+}$, respectively, then

$$
\left\|(u(t)-\bar{u}(t))^{+}\right\|_{1} \leq\left\|\left(u_{0}-\bar{u}_{0}\right)^{+}\right\|_{1} \quad \text { for all } t \geq 0 .
$$

Proof. Existence of entropy solutions. Given $0 \leq u_{0} \in L^{\infty}\left(\mathbb{R}^{N}\right) \cap L^{1}\left(\mathbb{R}^{N}\right)$, let $u(t)=$ $T(t) u_{0}$, where $(T(t))_{t \geq 0}$ is the semigroup in $L^{1}\left(\mathbb{R}^{N}\right)^{+}$generated by the accretive operator $\mathcal{B}$. Then, according to the general theory of nonlinear semigroups ([11]), $u(t)$ is a mild solution of the abstract Cauchy problem

$$
u^{\prime}(t)+B u(t) \ni 0, \quad u(0)=u_{0} .
$$

Let us prove that $u$ is an entropy solution of 1.1 in $Q_{T}$. We divide the proof into several steps. 
Step 1. Approximation with Crandall-Liggett's scheme. Let $T>0, K \geq 1, \Delta t=T / K$, $t_{n}=n \Delta t, n=0, \ldots, K-1$. We define inductively $u^{n+1}, n=0, \ldots, K$, to be the unique entropy solution of

$$
\frac{u^{n+1}-u^{n}}{\Delta t}-\operatorname{div} \mathbf{a}\left(u^{n+1}, D u^{n+1}\right)=0 \quad \text { in } \mathbb{R}^{N}
$$

where $u^{0}=u_{0}$. Recall that $\left\|u^{n}\right\|_{q} \leq\left\|u_{0}\right\|_{q}$ for all $n \in \mathbb{N}$ and $q=1, \infty$. We define

$$
u^{K}(t)=u^{0} \chi_{\left[t_{0}, t_{1}\right]}(t)+\sum_{n=1}^{K-1} u^{n} \chi_{\left(t_{n}, t_{n+1}\right]}(t) .
$$

We know that $u^{K}$ converges uniformly to $u \in C\left([0, T], L^{1}\left(\mathbb{R}^{N}\right)\right)$ and

$$
\|u(t)\|_{p} \leq\left\|u_{0}\right\|_{p} \quad \forall p \in[1, \infty] .
$$

Define

$$
\xi^{K}(t):=\sum_{n=0}^{K-1} \frac{u^{n+1}-u^{n}}{\Delta t} \chi_{\left(t_{n}, t_{n+1}\right]}(t)
$$

and

$$
\mathbf{z}^{K}(t)=\mathbf{a}\left(u^{1}, \nabla u^{1}\right) \chi_{\left[t_{0}, t_{1}\right]}(t)+\sum_{n=1}^{K-1} \mathbf{a}\left(u^{n+1}, \nabla u^{n+1}\right) \chi_{\left(t_{n}, t_{n+1}\right]}(t) .
$$

Since $u^{n+1}$ is the entropy solution of 4.5 , we have

$$
\xi^{K}(t)=\operatorname{div}\left(\mathbf{z}^{K}(t)\right) \quad \text { in } \mathcal{D}^{\prime}\left(\mathbb{R}^{N}\right)
$$

and for all $S, T \in \mathcal{T}^{+}$, we have

$h_{S}\left(u^{K}(t+\Delta t), D T\left(u^{K}(t+\Delta t)\right)\right) \leq\left(\mathbf{z}^{K}(t), D J_{T^{\prime} S}\left(u^{K}(t+\Delta t)\right)\right)$ as measures,

$h\left(u^{K}(t+\Delta t), D T\left(u^{K}(t+\Delta t)\right)\right) \leq\left(\mathbf{z}^{K}(t), D T\left(u^{K}(t+\Delta t)\right)\right) \quad$ as measures.

Since $\left\|\mathbf{z}^{K}(t)\right\|_{\infty} \leq M$ for all $K \in \mathbb{N}$ and a.e. $t \in[0, T]$, we may assume that

$$
\mathbf{z}^{K} \rightarrow \mathbf{z} \in L^{\infty}\left(Q_{T}, \mathbb{R}^{N}\right) \quad \text { weak* }
$$

Moreover, since $\mathbf{z}^{K}(t)=u^{K}(t+\Delta t) \mathbf{b}\left(\nabla u^{K}(t+\Delta t)\right)$ with $\| \mathbf{b}\left(\nabla u^{K}(t+\Delta t) \| \leq M_{0}\right.$ (where the constant $M_{0}$ is independent of $K$ and $t$ ) and $u^{K}$ converges uniformly to $u$ in $C\left([0, T], L^{1}\left(\mathbb{R}^{N}\right)\right)$, we may also assume that

$$
\mathbf{b}\left(\nabla u^{K}(t+\Delta t)\right) \rightarrow \mathbf{z}_{\mathbf{b}}(t) \in L^{\infty}\left(Q_{T}, \mathbb{R}^{N}\right) \quad \text { weak }^{*}
$$

and

$$
\mathbf{z}(t)=u(t) \mathbf{z}_{\mathbf{b}}(t) \quad \text { for almost all } t \in[0, T] .
$$


Step 2. Working as in the proof of Theorem 3 of [5], we can prove the following facts.

Lemma 4.6. We have

$$
\xi=\operatorname{div}_{x}(\mathbf{z}) \quad \text { in } \mathcal{D}^{\prime}\left(Q_{T}\right)
$$

Moreover

$$
\xi(t)=\operatorname{div}_{x}(\mathbf{z}(t)) \quad \text { in } \mathcal{D}^{\prime}\left(\mathbb{R}^{N}\right) \quad \text { for a.e. } t \in[0, T] .
$$

Hence, $(\mathbf{z}(t), \xi(t)) \in Z\left(\mathbb{R}^{N}\right)$ for almost all $t \in[0, T]$.

Lemma 4.7. $\xi$ is the time derivative of $u$ in the sense of Definition 4.2

Lemma 4.8. $\xi=\operatorname{div}(\mathbf{z})$ in $\left(L^{1}\left(0, T, \mathrm{BV}\left(\mathbb{R}^{N}\right)_{2}\right)\right)^{*}$ in the sense of Definition 4.3

Step 3. Some auxiliary inequalities. Let $\mathcal{M}\left(\mathbb{R}^{N}\right)$ be the space of Radon measures in $\mathbb{R}^{N}$. Fix $p=T_{a, b} \in \mathcal{T}_{r}$, and let $j$ be the primitive of $p$. Working as in the proof of Step 5 of Theorem 3 in [5], we obtain the following result.

Lemma 4.9. We have

$$
\int_{0}^{T} \int_{\mathbb{R}^{N}}\left(\mathbf{z}^{K}(t), D\left(p\left(u^{K}(t+\Delta t)\right) \phi\right)\right) d t \leq \int_{0}^{T} \int_{\mathbb{R}^{N}} j\left(u^{K}(t)\right) \frac{\phi(t)-\phi(t-\Delta t)}{\Delta t} d x d t
$$

for any $0 \leq \phi \in \mathcal{D}\left((0, T) \times \mathbb{R}^{N}\right)$. The same inequality holds for any $0 \leq \phi \in \mathcal{D}((0, T))$. As a consequence,

$$
\left\{\left(\mathbf{z}^{K}(t), D p\left(u^{K}(t+\Delta t)\right)\right)\right\} \quad \text { is a bounded sequence in } L_{\text {loc }}^{1}\left((0, T), \mathcal{M}\left(\mathbb{R}^{N}\right)\right) \text {. }
$$

Moreover, $p(u(\cdot)) \in L_{\text {loc }, w}^{1}\left(0, T, \mathrm{BV}\left(\mathbb{R}^{N}\right)\right)$.

By (4.15), by extracting a subsequence if necessary we may assume that there is $\mu_{p} \in$ $\mathcal{M}\left(Q_{T}\right)$ such that

$$
\lim _{K} \int_{0}^{T} \int_{\mathbb{R}^{N}} \phi\left(\mathbf{z}^{K}(t), D p\left(u^{K}(t+\Delta t)\right)\right)=\left\langle\mu_{p}, \phi\right\rangle \quad \forall \phi \in C_{c}\left(Q_{T}\right) .
$$

Let $0 \leq \phi \in \mathcal{D}\left((0, T) \times \mathbb{R}^{N}\right)$. Writing 4.14 as

$$
\begin{aligned}
\int_{0}^{T} \int_{\mathbb{R}^{N}} \phi\left(\mathbf{z}^{K}(t), D p\left(u^{K}(t\right.\right. & +\Delta t))) d t \\
\leq & \int_{0}^{T} \int_{\mathbb{R}^{N}} j\left(u^{K}(t)\right) \frac{\phi(t)-\phi(t-\Delta t)}{\Delta t} d x d t \\
& -\int_{0}^{T} \int_{\mathbb{R}^{N}} \mathbf{z}^{K}(t) \cdot \nabla \phi p\left(u^{K}(t+\Delta t)\right) d x d t
\end{aligned}
$$

and letting $K \rightarrow \infty$ we obtain

$$
\left\langle\mu_{p}, \phi\right\rangle \leq \int_{0}^{T} \int_{\mathbb{R}^{N}} j(u(t)) \phi^{\prime}(t) d x d t-\int_{0}^{T} \int_{\mathbb{R}^{N}} \mathbf{z}(t) \cdot \nabla \phi p(u(t)) d x d t .
$$


Fix $S, T \in \mathcal{T}^{+}$and $0 \leq \phi \in \mathcal{D}\left(Q_{T}\right)$. Working as in the proof of Step 5 of Theorem 3 in [5] we obtain both the analogue of 4.16,

$$
\begin{aligned}
\int_{0}^{T} \int_{\mathbb{R}^{N}} \phi\left(\mathbf{z}^{K}(t), D\left(T\left(u^{K}(t+\Delta t) S\left(u^{K}(t+\Delta t)\right)\right)\right) d t\right. \\
\leq \int_{0}^{T} \int_{\mathbb{R}^{N}} J_{T S}\left(u^{K}(t)\right) \frac{\phi(t)-\phi(t-\Delta t)}{\Delta t} d x d t \\
\quad-\int_{0}^{T} \int_{\mathbb{R}^{N}} \mathbf{z}^{K}(t) \cdot \nabla \phi S\left(u^{K}(t+\Delta t)\right) T\left(u^{K}(t+\Delta t)\right) d x d t
\end{aligned}
$$

and the fact that

$$
\left\{\left(\mathbf{z}^{K}(t), D\left(T\left(u^{K}(t+\Delta t) S\left(u^{K}(t+\Delta t)\right)\right)\right)\right\}\right.
$$

is a bounded sequence in $L_{\text {loc }}^{1}\left((0, T), \mathcal{M}\left(\mathbb{R}^{N}\right)\right)$. Now, by 3.17), we have

$$
\begin{aligned}
\left(\mathbf{z}^{K}(t), D\left(T\left(u^{K}(t+\Delta t) S\left(u^{K}(t+\Delta t)\right)\right)\right)\right) \\
\quad=\left(\mathbf{z}^{K}(t), D J_{T^{\prime} S}\left(u^{K}(t+\Delta t)\right)\right)+\left(\mathbf{z}^{K}(t), D J_{S^{\prime} T}\left(u^{K}(t+\Delta t)\right)\right),
\end{aligned}
$$

and, by 4.8 , the measures $\left(\mathbf{z}^{K}(t), D J_{T^{\prime} S}\left(u^{K}(t+\Delta t)\right)\right)$ and $\left(\mathbf{z}^{K}(t), D J_{S^{\prime} T}\left(u^{K}(t+\Delta t)\right)\right)$ are positive. Hence

$$
\left\{\left(\mathbf{z}^{K}(t), D J_{T^{\prime} S}\left(u^{K}(t+\Delta t)\right)\right)\right\} \quad \text { and } \quad\left\{\left(\mathbf{z}^{K}(t), D J_{S^{\prime} T}\left(u^{K}(t+\Delta t)\right)\right)\right\}
$$

are bounded sequences in $L_{\text {loc }}^{1}\left((0, T), \mathcal{M}\left(\mathbb{R}^{N}\right)\right)$. By extracting a subsequence if necessary, we may assume that there exist $\mu_{S}^{T}, \mu_{T}^{S} \in \mathcal{M}\left(Q_{T}\right)$ such that

$$
\lim _{K} \int_{0}^{T} \int_{\mathbb{R}^{N}} \phi\left(\mathbf{z}^{K}(t), D J_{T^{\prime} S}\left(u^{K}(t+\Delta t)\right)\right)=\left\langle\mu_{S}^{T}, \phi\right\rangle, \quad \forall \phi \in C_{c}\left(Q_{T}\right),
$$

and

$$
\lim _{K} \int_{0}^{T} \int_{\mathbb{R}^{N}} \phi\left(\mathbf{z}^{K}(t), D J_{S^{\prime} T}\left(u^{K}(t+\Delta t)\right)\right)=\left\langle\mu_{T}^{S}, \phi\right\rangle, \quad \forall \phi \in C_{c}\left(Q_{T}\right) .
$$

Now, passing to the limit in (4.18), and using (4.19) and (4.20), we obtain

$$
\begin{aligned}
\left\langle\mu_{S}^{T}, \phi\right\rangle+\left\langle\mu_{T}^{S}, \phi\right\rangle \leq & \int_{0}^{T} \int_{\mathbb{R}^{N}} J_{T S}(u(t)) \phi^{\prime}(t) d x d t \\
& -\int_{0}^{T} \int_{\mathbb{R}^{N}} \mathbf{z}(t) \cdot \nabla \phi T(u(t)) S(u(t)) d x d t,
\end{aligned}
$$

and this holds for all $\phi \in \mathcal{D}\left(Q_{T}\right)$.

Step 5. Identification of the vector field. Let us now prove that

$$
\mathbf{z}(t)=\mathbf{a}(u(t), \nabla u(t)) \quad \text { for a.e. } t \in(0, T) .
$$

We use Minty-Browder's technique. Let $0<a<b$, let $0 \leq \phi \in C_{0}^{1}\left((0, T) \times \mathbb{R}^{N}\right)$ and $g \in C^{2}\left(\mathbb{R}^{N}\right) \cap W^{1, \infty}\left(\mathbb{R}^{N}\right)$. Let

$$
J_{\mathbf{a}_{i}}(x, r):=\int_{0}^{r} \mathbf{a}_{i}(s, \nabla g(x)) d s, \quad J_{\partial \mathbf{a}_{i} / \partial x_{j}}(x, r):=\int_{0}^{r} \frac{\partial}{\partial x_{j}} \mathbf{a}_{i}(s, \nabla g(x)) d s,
$$


for $i, j \in\{1, \ldots, N\}$. For simplicity, write

$$
\begin{aligned}
& D_{2} J_{\mathbf{a}}\left(x, T_{a, b}\left(u^{K}(t+\Delta t)\right)\right) \\
& :=\sum_{i=1}^{N}\left[\frac{\partial}{\partial x_{i}} J_{\mathbf{a}_{i}}\left(x, T_{a, b}\left(u^{K}(t+\Delta t)\right)\right)-J_{\partial \mathbf{a}_{i} / \partial x_{i}}\left(x, T_{a, b}\left(u^{K}(t+\Delta t)\right)\right)\right] .
\end{aligned}
$$

Let us make some remarks concerning the measure $D_{2} J_{\mathbf{a}}\left(x, T_{a, b}\left(u^{K}(t+\Delta t)\right)\right)$. Using Volpert's averaged superposition

$$
\begin{aligned}
& \overline{\mathbf{a}}\left(T_{a, b}\left(u^{K}(t+\Delta t)\right), \nabla g(x)\right) \\
& \quad=\int_{0}^{1} \mathbf{a}\left(\tau\left(T_{a, b}\left(u^{K}(t+\Delta t)\right)\right)^{+}+(1-\tau)\left(T_{a, b}\left(u^{K}(t+\Delta t)\right)\right)^{-}, \nabla g(x)\right) d \tau,
\end{aligned}
$$

and the chain rule for BV functions ([1, Theorem 3.96]), and working as we did in the proof of Theorem 3.6 (see [6]), we obtain

$$
\begin{aligned}
D_{2} J_{\mathbf{a}}\left(x, T_{a, b}\left(u^{K}(t+\Delta t)\right)\right)= & \mathbf{a}\left(T_{a, b}\left(u^{K}(t+\Delta t)\right), \nabla g\right) \cdot \nabla T_{a, b}\left(u^{K}(t+\Delta t)\right) \\
& +\overline{\mathbf{a}}\left(T_{a, b}\left(u^{K}(t+\Delta t)\right), \nabla g(x)\right) \cdot D^{s} T_{a, b}\left(u^{K}(t+\Delta t)\right) .
\end{aligned}
$$

In particular, we observe that the absolutely continuous part of $D_{2} J_{\mathbf{a}}\left(x, T_{a, b}\left(u^{K}(t+\Delta t)\right)\right)$ is

$$
\begin{array}{r}
\mathbf{a}\left(T_{a, b}\left(u^{K}(t+\Delta t)\right), \nabla g\right) \cdot \nabla T_{a, b}\left(u^{K}(t+\Delta t)\right) \mathcal{L}^{N} \\
=\mathbf{a}\left(u^{K}(t+\Delta t), \nabla g\right) \cdot \nabla T_{a, b}\left(u^{K}(t+\Delta t)\right) \mathcal{L}^{N} .
\end{array}
$$

Using (4.9) and 3.4], after some calculation we get

$$
\begin{aligned}
& \int_{0}^{T} \int_{\mathbb{R}^{N}} \phi\left(\mathbf{z}^{K}(t), D T_{a, b}\left(u^{K}(t+\Delta t)-\nabla g\right)\right) d t \\
& \quad-\int_{0}^{T} \int_{\mathbb{R}^{N}} \phi\left[D_{2} J_{\mathbf{a}}\left(x, T_{a, b}\left(u^{K}(t+\Delta t)\right)\right)-\mathbf{a}\left(u^{K}(t+\Delta t), \nabla g\right) \cdot \nabla g\right] d t \\
& \geq-\int_{0}^{T} \int_{\mathbb{R}^{N}} \phi \mathbf{z}^{K}(t) \cdot \nabla g\left(1-T_{a, b}^{\prime}\left(u^{K}(t+\Delta t)\right)\right) d x d t \\
& +\int_{0}^{T} \int_{\mathbb{R}^{N}} \phi\left[h \left(T _ { a , b } \left(u^{K}(t+\Delta t), D T_{a, b}\left(u^{K}(t+\Delta t)\right)^{s}-\left(D_{2} J_{\mathbf{a}}\left(x, T_{a, b}\left(u^{K}(t+\Delta t)\right)\right)^{s}\right] d t .\right.\right.\right.
\end{aligned}
$$

On the other hand, by $\left(\mathrm{H}_{5}\right),\left(\mathrm{H}_{6}\right)$ and using the chain rule for BV functions, it is not difficult to prove that

$$
\left(D_{2} J_{\mathbf{a}}\left(x, T_{a, b}\left(u^{K}(t+\Delta t)\right)\right)\right)^{s}=h\left(T_{a, b}\left(u^{K}(t+\Delta t)\right), D T_{a, b}\left(u^{K}(t+\Delta t)\right)\right)^{s} .
$$


Consequently,

$$
\begin{aligned}
& \int_{0}^{T} \int_{\mathbb{R}^{N}} \phi\left[h\left(T_{a, b}\left(u^{K}(t+\Delta t)\right), D T_{a, b}\left(u^{K}(t+\Delta t)\right)\right)^{s}\right. \\
&\left.-\left(D_{2} J_{\mathbf{a}}\left(x, T_{a, b}\left(u^{K}(t+\Delta t)\right)\right)\right)^{s}\right] d t \geq 0 .
\end{aligned}
$$

Moreover, we have

$$
\begin{aligned}
\int_{0}^{T} \int_{\mathbb{R}^{N}} \phi \mathbf{z}^{K}(t) & \cdot \nabla g\left(1-T_{a, b}^{\prime}\left(u^{K}(t+\Delta t)\right)\right) d x d t \\
\leq & \int_{0}^{T} \int_{\mathbb{R}^{N}} \phi \mathbf{z}^{K}(t) \cdot \nabla g\left(1-T_{a, b}^{\prime}\left(u^{K}(t+\Delta t)\right)\right) T_{a, b}^{\prime}(u(t)) d x d t \\
& +M\|\nabla g\|_{\infty} \int_{0}^{T} \int_{\mathbb{R}^{N}} \phi\left(1-T_{a, b}^{\prime}(u(t))\right) d x d t .
\end{aligned}
$$

Therefore, we obtain

$$
\begin{aligned}
& \int_{0}^{T} \int_{\mathbb{R}^{N}} \phi\left(\mathbf{z}^{K}(t), D\left(T_{a, b}\left(u^{K}(t+\Delta t)\right)-g\right)\right) d t \\
& \quad-\int_{0}^{T} \int_{\mathbb{R}^{N}} \phi\left[D_{2} J_{\mathbf{a}}\left(x, T_{a, b}\left(u^{K}(t+\Delta t)\right)\right)-\mathbf{a}\left(u^{K}(t+\Delta t), \nabla g\right) \cdot \nabla g\right] d t \\
& \quad+\int_{0}^{T} \int_{\mathbb{R}^{N}} \phi \mathbf{z}^{K}(t) \cdot \nabla g\left(1-T_{a, b}^{\prime}\left(u^{K}(t+\Delta t)\right)\right) T_{a, b}^{\prime}(u(t)) d x d t \\
& \quad+M\|\nabla g\|_{\infty} \int_{0}^{T} \int_{\mathbb{R}^{N}} \phi\left(1-T_{a, b}^{\prime}(u(t))\right) d x d t \geq 0 .
\end{aligned}
$$

Our purpose is to take limits as $K \rightarrow \infty$ in the above inequality. We assume that $\phi(t, x)=\eta(t) \rho(x)$, where $\eta \in \mathcal{D}((0, T)), \rho \in \mathcal{D}\left(\mathbb{R}^{N}\right), \eta \geq 0, \rho \geq 0$. Let $j$ denote the primitive of $T_{a, b}$. First, integrating by parts in the first term, for $\Delta t$ small enough we have

$$
\begin{aligned}
& \int_{0}^{T} \int_{\mathbb{R}^{N}} \phi\left(\mathbf{z}^{K}(t), D\left(T_{a, b}\left(u^{K}(t+\Delta t)\right)-g\right)\right) d t \\
&=-\int_{0}^{T} \int_{\mathbb{R}^{N}}\left(T_{a, b}\left(u^{K}(t+\Delta t)\right)-g\right) \mathbf{z}^{K}(t) \cdot \nabla_{x} \phi(t) d x d t \\
&-\int_{0}^{T} \int_{\mathbb{R}^{N}} \phi(t)\left(T_{a, b}\left(u^{K}(t+\Delta t)\right)-g\right) \operatorname{div}\left(\mathbf{z}^{K}(t)\right) d x d t \\
&=-\int_{0}^{T} \int_{\mathbb{R}^{N}}\left(T_{a, b}\left(u^{K}(t+\Delta t)\right)-g\right) \mathbf{z}^{K}(t) \cdot \nabla_{x} \phi(t) d x d t \\
&-\int_{0}^{T} \int_{\mathbb{R}^{N}} \phi(t)\left(T_{a, b}\left(u^{K}(t+\Delta t)\right)-g\right) \xi^{K}(t) d x d t .
\end{aligned}
$$


Now,

$$
\begin{aligned}
\int_{0}^{T} \int_{\mathbb{R}^{N}} \phi(t) & \left(T_{a, b}\left(u^{K}(t+\Delta t)\right)-g\right) \xi^{K}(t) d x d t \\
= & \int_{0}^{T} \int_{\mathbb{R}^{N}} \phi(t) T_{a, b}\left(u^{K}(t+\Delta t)\right) \frac{u^{K}(t+\Delta t)-u^{K}(t)}{\Delta t} d t \\
& -\int_{0}^{T} \int_{\mathbb{R}^{N}} \phi(t) g \xi^{K}(t) d t \\
\geq & \int_{0}^{T} \int_{\mathbb{R}^{N}} \phi(t) \frac{j\left(u^{K}(t+\Delta t)\right)-j\left(u^{K}(t)\right)}{\Delta t} d t-\int_{0}^{T} \int_{\mathbb{R}^{N}} \phi(t) g \xi^{K}(t) d t \\
= & \int_{0}^{T} \int_{\mathbb{R}^{N}} \frac{\phi(t-\Delta t)-\phi(t)}{\Delta t} j\left(u^{K}(t)\right) d t-\int_{0}^{T} \int_{\mathbb{R}^{N}} \phi(t) g \xi^{K}(t) d t .
\end{aligned}
$$

Hence,

$$
\begin{aligned}
& \int_{0}^{T} \int_{\mathbb{R}^{N}} \phi\left(\mathbf{z}^{K}(t), D\left(u^{K}(t+\Delta t)-g\right)\right) \\
& \leq-\int_{0}^{T} \int_{\mathbb{R}^{N}} \frac{\phi(t-\Delta t)-\phi(t)}{\Delta t} j\left(u^{K}(t)\right) d t \\
&+\int_{0}^{T} \int_{\mathbb{R}^{N}} \phi(t) g \xi^{K}(t) d t \\
&-\int_{0}^{T} \int_{\mathbb{R}^{N}}\left(T_{a, b}\left(u^{K}(t+\Delta t)\right)-g\right) \mathbf{z}^{K}(t) \cdot \nabla_{x} \phi(t) d x d t .
\end{aligned}
$$

Then from 4.24) it follows that

$$
\begin{aligned}
-\int_{0}^{T} & \int_{\mathbb{R}^{N}} \frac{\phi(t-\Delta t)-\phi(t)}{\Delta t} j\left(u^{K}(t)\right) d t+\int_{0}^{T} \int_{\mathbb{R}^{N}} \phi(t) g \xi^{K}(t) d t \\
& -\int_{0}^{T} \int_{\mathbb{R}^{N}}\left(T_{a, b}\left(u^{K}(t+\Delta t)\right)-g\right) \mathbf{z}^{K}(t) \cdot \nabla_{x} \phi(t) d x d t \\
& +\int_{0}^{T} \int_{\mathbb{R}^{N}} \phi\left[-D_{2} J_{\mathbf{a}}\left(x, T_{a, b}\left(u^{K}(t+\Delta t)\right)\right)+\mathbf{a}\left(u^{K}(t+\Delta t), \nabla g\right) \cdot \nabla g\right] d t \\
& +\int_{0}^{T} \int_{\mathbb{R}^{N}} \phi \mathbf{z}^{K}(t) \cdot \nabla g\left(1-T_{a, b}^{\prime}\left(u^{K}(t+\Delta t)\right)\right) T_{a, b}^{\prime}(u(t)) d x d t \\
& +M\|\nabla g\|_{\infty} \int_{0}^{T} \int_{\mathbb{R}^{N}} \phi\left(1-T_{a, b}^{\prime}(u(t))\right) d x d t \geq 0 .
\end{aligned}
$$

Letting $K \rightarrow \infty$ in 4.25 , observing that the integral in the next to last line goes to zero, and having in mind that

$$
D_{2} J_{\mathbf{a}}\left(x, T_{a, b}\left(u^{K}(t+\Delta t)\right)\right) \rightarrow D_{2} J_{\mathbf{a}}\left(x, T_{a, b}(u(t+\Delta t))\right) \quad \text { weakly as measures, }
$$


working as in the proof of Theorem 3.6 (see [6] and also [4]), we obtain

$$
\begin{aligned}
\int_{0}^{T} \int_{\mathbb{R}^{N}} \phi^{\prime}(t) j(u(t)) d t & +\int_{0}^{T}\langle\phi(t) g, \xi(t)\rangle d t \\
& -\int_{0}^{T} \int_{\mathbb{R}^{N}}\left(T_{a, b}(u(t))-g\right) \mathbf{z}(t) \cdot \nabla_{x} \phi(t) d x d t \\
& +\int_{0}^{T} \int_{\mathbb{R}^{N}} \phi\left[-D_{2} J_{\mathbf{a}}\left(x, T_{a, b}(u(t))\right)+\mathbf{a}(u(t), \nabla g) \cdot \nabla g\right] d t \\
& +M\|\nabla g\|_{\infty} \int_{0}^{T} \int_{\mathbb{R}^{N}} \phi\left(1-T_{a, b}^{\prime}(u(t))\right) d x d t \geq 0 .
\end{aligned}
$$

Now, using Lemma 4.8 , we get

$$
\begin{aligned}
\int_{0}^{T} \int_{\mathbb{R}^{N}} \phi^{\prime}(t) j(u(t)) d t-\int_{0}^{T} \int_{\mathbb{R}^{N}} \phi(t) \mathbf{z}(t) \cdot \nabla g d x d t \\
-\int_{0}^{T} \int_{\mathbb{R}^{N}} T_{a, b}(u(t)) \mathbf{z}(t) \cdot \nabla_{x} \phi(t) d x d t \\
+\int_{0}^{T} \int_{\mathbb{R}^{N}} \phi\left[-D_{2} J_{\mathbf{a}}\left(x, T_{a, b}(u(t))\right)+\mathbf{a}(u(t), \nabla g) \cdot \nabla g\right] d t \\
+M\|\nabla g\|_{\infty} \int_{0}^{T} \int_{\mathbb{R}^{N}} \phi\left(1-T_{a, b}^{\prime}(u(t))\right) d x d t \geq 0 .
\end{aligned}
$$

For any $\tau>0$, we define the function $\eta^{\tau}$ as the Dunford integral (see [21])

$$
\eta^{\tau}(t):=\frac{1}{\tau} \int_{t-\tau}^{t} \eta(s) T_{a, b}(u(s)) d s \in \mathrm{BV}\left(\mathbb{R}^{N}\right)^{* *}
$$

Using this function as in the proof of Theorem 3 of [5], we obtain the following result.

Lemma 4.10. We have

$$
\begin{aligned}
\int_{0}^{T} \int_{\mathbb{R}^{N}} \phi^{\prime}(t) j(u(t)) d t \leq & \int_{0}^{T} \eta(t) \int_{\mathbb{R}^{N}} T_{a, b}(u(t)) \mathbf{z}(t) \cdot \nabla_{x} \rho d x d t \\
& +\int_{0}^{T} \eta(t) \int_{\mathbb{R}^{N}} \rho \mathbf{z}(t) \cdot \nabla T_{a, b}(u(t)) d x d t \\
& +\int_{0}^{T} \eta(t) \int_{\mathbb{R}^{N}} \rho M\left|D^{s}\left(T_{a, b}(u(t))\right)\right| d t
\end{aligned}
$$


Now, we may conclude the proof of 4.22). Using Lemma 4.10, and taking into account 4.26, we obtain

$$
\begin{aligned}
& 0 \leq \int_{0}^{T} \eta(t) \int_{\mathbb{R}^{N}} T_{a, b}(u(t)) \mathbf{z}(t) \cdot \nabla_{x} \rho d x d t \\
& +\int_{0}^{T} \eta(t) \int_{\mathbb{R}^{N}} \rho \mathbf{z}(t) \cdot \nabla T_{a, b}(u(t)) d x d t+\int_{0}^{T} \eta(t) \int_{\mathbb{R}^{N}} \rho M\left|D^{S}\left(T_{a, b}(u(t))\right)\right| d t \\
& -\int_{0}^{T} \int_{\mathbb{R}^{N}} \phi(t) \mathbf{z}(t) \cdot \nabla g d x d t-\int_{0}^{T} \int_{\mathbb{R}^{N}} T_{a, b}(u(t)) \mathbf{z}(t) \cdot \nabla_{x} \phi(t) d x d t \\
& +\int_{0}^{T} \int_{\mathbb{R}^{N}} \phi\left[-D_{2} J_{\mathbf{a}}\left(x, T_{a, b}(u(t))\right)+\mathbf{a}(u(t), \nabla g) \cdot \nabla g\right] d t \\
& +M\|\nabla g\|_{\infty} \int_{0}^{T} \int_{\mathbb{R}^{N}} \phi\left(1-T_{a, b}^{\prime}(u(t))\right) d x d t \\
& =\int_{0}^{T} \eta(t) \int_{\mathbb{R}^{N}} \rho \mathbf{z}(t) \cdot \nabla T_{a, b}(u(t)) d x d t \\
& +\int_{0}^{T} \eta(t) \int_{\mathbb{R}^{N}} \rho M\left|D^{s}\left(T_{a, b}(u(t))\right)\right| d t-\int_{0}^{T} \eta(t) \int_{\mathbb{R}^{N}} \rho(x) \mathbf{z}(t) \cdot \nabla g d x d t \\
& +\int_{0}^{T} \eta(t) \int_{\mathbb{R}^{N}} \rho(x)\left[-\mathbf{a}(u, \nabla g) \cdot\left(\nabla T_{a, b}(u(t))-\nabla g\right)\right] d x d t \\
& -\int_{0}^{T} \eta(t) \int_{\mathbb{R}^{N}} \rho\left(D_{2} J_{\mathbf{a}}\left(x, T_{a, b}(u(t))\right)\right)^{s} d t \\
& +M\|\nabla g\|_{\infty} \int_{0}^{T} \int_{\mathbb{R}^{N}} \phi\left(1-T_{a, b}^{\prime}(u(t))\right) d x d t \\
& =\int_{0}^{T} \eta(t) \int_{\mathbb{R}^{N}} \rho(x)(\mathbf{z}(t)-\mathbf{a}(u(t), \nabla g)) \cdot\left(\nabla T_{a, b}(u(t))-\nabla g\right) d x d t \\
& +\int_{0}^{T} \eta(t) \int_{\mathbb{R}^{N}} \rho\left(M\left|D^{s}\left(T_{a, b}(u(t))\right)\right|-\left(D_{2} J_{\mathbf{a}}(x, u(t))\right)^{s}\right) d t \\
& +M\|\nabla g\|_{\infty} \int_{0}^{T} \int_{\mathbb{R}^{N}} \phi\left(1-T_{a, b}^{\prime}(u(t))\right) d x d t
\end{aligned}
$$

for all $\phi(t, x)=\eta(t) \rho(x), \eta \in \mathcal{D}((0, T)), \rho \in \mathcal{D}\left(\mathbb{R}^{N}\right), \eta, \rho \geq 0$. Thus, the measure

$$
\begin{aligned}
{[\mathbf{z}(t)-\mathbf{a}(u(t), \nabla g)] } & \cdot \nabla\left(T_{a, b}(u(t))-g\right)+M\left|D^{s}\left(T_{a, b}(u(t))\right)\right| \\
& -\left(D_{2} J_{\mathbf{a}}\left(x, T_{a, b}(u(t))\right)\right)^{s}+M\|\nabla g\|_{\infty}\left(1-T_{a, b}^{\prime}(u(t))\right) \geq 0 .
\end{aligned}
$$

Then its absolutely continuous part is

$$
[\mathbf{z}(t)-\mathbf{a}(u(t), \nabla g)] \cdot \nabla\left(T_{a, b}(u(t))-g\right)+M\|\nabla g\|_{\infty}\left(1-T_{a, b}^{\prime}(u(t))\right) \geq 0 .
$$

In particular, we have

$$
[\mathbf{z}(t)-\mathbf{a}(u(t), \nabla g)] \cdot \nabla(u(t)-g) \geq 0 \quad \text { a.e. on }[a<u<b] .
$$


Since we may take a countable set in $C^{2}\left(\mathbb{R}^{N}\right) \cap W^{1, \infty}\left(\mathbb{R}^{N}\right)$ which is dense in $C^{1}\left(\mathbb{R}^{N}\right)$, the above inequality holds for all $(t, x) \in S \cap[a<u<b]$ where $S \subseteq(0, T) \times \mathbb{R}^{N}$ is such that $\mathcal{L}^{N}\left((0, T) \times \mathbb{R}^{N} \backslash S\right)=0$, and all $g \in C^{1}\left(\mathbb{R}^{N}\right)$. Now, fix $(t, x) \in S \cap[a<u<b]$. Given $y \in \mathbb{R}^{N}$, there is $g \in C^{1}\left(\mathbb{R}^{N}\right)$ such that $\nabla g(x)=y$. Then

$$
(\mathbf{z}(t, x)-\mathbf{a}(u(t), y)) \cdot(\nabla u(t, x)-y) \geq 0 \quad \forall y \in \mathbb{R}^{N}, \forall(t, x) \in S \cap[a<u<b] .
$$

By an application of Minty-Browder's method in $\mathbb{R}^{N}$, it follows that

$$
\mathbf{z}(t, x)=\mathbf{a}(u(t, x), \nabla u(t, x)) \quad \text { for a.e. }(t, x) \in Q_{T} \cap[a<u<b] .
$$

Since this holds for any $0<a<b$, we obtain 4.22 at a.e. point $(t, x)$ of $Q_{T}$ such that $u(t, x) \neq 0$. Now, by our assumptions on $\mathbf{a}$ and 4.11) we deduce that $\mathbf{z}(t, x)=$ $\mathbf{a}(u(t, x), \nabla u(t, x))=0$ a.e. on $[u=0]$. We have proved (4.22).

Step 6. A final lemma and conclusion

Lemma 4.11. For the functions $S, T \in \mathcal{T}^{+}$used above, we have

$$
\mu_{S}^{T} \geq h_{S}(u, D T(u)) .
$$

Proof. By (4.8), we have

$$
\mathbf{z}^{K}(t) \cdot D^{S} J_{T^{\prime} S}\left(u^{K}(t+\Delta t)\right) \geq f_{S}\left(u^{K}(t+\Delta t), D T\left(u^{K}(t+\Delta t)\right)\right)^{s}
$$

for all $t \in(0, T)$. Let $0 \leq \phi \in C_{c}\left(Q_{T}\right)$. Using this inequality and the convexity of $f$, we compute

$$
\begin{aligned}
\int_{0}^{T} \int_{\mathbb{R}^{N}} \phi S\left(u^{K}(t+\Delta t)\right) \mathbf{a}\left(u^{K}(t+\Delta t), \nabla T\left(u^{K}(t+\Delta t)\right) \cdot \nabla T(u(t))\right) d x d t \\
\leq \quad \int_{0}^{T} \int_{\mathbb{R}^{N}} \phi S\left(u^{K}(t+\Delta t)\right) \mathbf{z}^{K}(t) \cdot \nabla T\left(u^{K}(t+\Delta t)\right) d x d t \\
\quad+\int_{0}^{T} \int_{\mathbb{R}^{N}} \phi S\left(u^{K}(t+\Delta t)\right) f\left(u^{K}(t+\Delta t), \nabla T(u)\right) d x d t \\
\quad-\int_{0}^{T} \int_{\mathbb{R}^{N}} \phi S\left(u^{K}(t+\Delta t)\right) f\left(u^{K}(t+\Delta t), \nabla T\left(u^{K}(t+\Delta t)\right)\right) d x d t \\
=\quad \int_{0}^{T} \int_{\mathbb{R}^{N}} \phi\left(\mathbf{z}^{K}(t), D J_{T^{\prime} S}\left(u^{K}(t+\Delta t)\right)\right) d t \\
\quad-\int_{0}^{T} \int_{\mathbb{R}^{N}} \phi \mathbf{z}^{K}(t) \cdot D^{s} J_{T^{\prime} S}\left(u^{K}(t+\Delta t)\right) d t \\
\quad+\int_{0}^{T} \int_{\mathbb{R}^{N}} \phi S\left(u^{K}(t+\Delta t)\right) f\left(u^{K}(t+\Delta t), \nabla T(u)\right) d x d t \\
\quad-\int_{0}^{T} \int_{\mathbb{R}^{N}} \phi S\left(u^{K}(t+\Delta t)\right) f\left(u^{K}(t+\Delta t), \nabla T\left(u^{K}(t+\Delta t)\right)\right) d x d t
\end{aligned}
$$




$$
\begin{aligned}
\leq & \int_{0}^{T} \int_{\mathbb{R}^{N}} \phi\left(\mathbf{z}^{K}(t), D J_{T^{\prime} S}\left(u^{K}(t+\Delta t)\right)\right) d t \\
& -\int_{0}^{T} \int_{\mathbb{R}^{N}} \phi f_{S}\left(u^{K}(t+\Delta t), D T\left(u^{K}(t+\Delta t)\right)\right)^{s} d t \\
& +\int_{0}^{T} \int_{\mathbb{R}^{N}} \phi S\left(u^{K}(t+\Delta t)\right) f\left(u^{K}(t+\Delta t), \nabla T(u)\right) d x d t \\
& -\int_{0}^{T} \int_{\mathbb{R}^{N}} \phi S\left(u^{K}(t+\Delta t)\right) f\left(u^{K}(t+\Delta t), \nabla T\left(u^{K}(t+\Delta t)\right)\right) d x d t \\
= & \int_{0}^{T} \int_{\mathbb{R}^{N}} \phi\left(\mathbf{z}^{K}(t), D J_{T^{\prime} S}\left(u^{K}(t+\Delta t)\right)\right) d t \\
& +\int_{0}^{T} \int_{\mathbb{R}^{N}} \phi S\left(u^{K}(t+\Delta t)\right) f\left(u^{K}(t+\Delta t), \nabla T(u)\right) d x d t \\
& -\int_{0}^{T} \int_{\mathbb{R}^{N}} \phi S\left(u^{K}(t+\Delta t)\right) f\left(u^{K}(t+\Delta t), D\left(u^{K}(t+\Delta t)\right)\right) d x d t .
\end{aligned}
$$

On the other hand,

$$
\begin{aligned}
& \int_{0}^{T} \int_{\mathbb{R}^{N}} \phi S\left(u^{K}(t+\Delta t)\right) \mathbf{z}^{K}(t) \cdot \nabla T(u(t)) d x d t \\
& =\int_{0}^{T} \int_{\mathbb{R}^{N}} \phi S\left(u^{K}(t+\Delta t)\right) \mathbf{a}\left(u^{K}(t+\Delta t), \nabla T\left(u^{K}(t+\Delta t)\right)\right) \cdot \nabla T(u(t)) d x d t+a_{K},
\end{aligned}
$$

with

$a_{K}:=$

$\int_{0}^{T} \int_{\mathbb{R}^{N}} \phi S\left(u^{K}(t+\Delta t)\right)\left(\mathbf{z}^{K}(t)-\mathbf{a}\left(u^{K}(t+\Delta t), \nabla T\left(u^{K}(t+\Delta t)\right)\right)\right) \cdot \nabla T(u(t)) d x d t$.

Hence,

$$
\begin{aligned}
\int_{0}^{T} & \int_{\mathbb{R}^{N}} \phi S\left(u^{K}(t+\Delta t)\right) \mathbf{z}^{K}(t) \cdot \nabla T(u(t)) d x d t \\
\leq & \int_{0}^{T} \int_{\mathbb{R}^{N}} \phi\left(\mathbf{z}^{K}(t), D J_{T^{\prime} S}\left(u^{K}(t+\Delta t)\right)\right) d t \\
& \quad+\int_{0}^{T} \int_{\mathbb{R}^{N}} \phi S\left(u^{K}(t+\Delta t)\right) f\left(u^{K}(t+\Delta t), \nabla T(u)\right) d x d t \\
& -\int_{0}^{T} \int_{\mathbb{R}^{N}} \phi S\left(u^{K}(t+\Delta t)\right) f\left(u^{K}(t+\Delta t), D\left(u^{K}(t+\Delta t)\right)\right) d x d t+a_{K} .
\end{aligned}
$$


Letting $K \rightarrow \infty$, using the fact that $\lim _{K \rightarrow+\infty} a_{K}=0$, 4.19), and

$$
\begin{aligned}
& \int_{0}^{T} \int_{\mathbb{R}^{N}} \phi f_{S}(u(t), D T(u(t))) d t \\
& \quad \leq \liminf _{K} \int_{0}^{T} \int_{\mathbb{R}^{N}} \phi S\left(u^{K}(t+\Delta t)\right) f\left(u^{K}(t+\Delta t), D\left(u^{K}(t+\Delta t)\right)\right) d t
\end{aligned}
$$

we obtain

$$
\begin{aligned}
\int_{0}^{T} \int_{\mathbb{R}^{N}} \phi S(u(t)) \mathbf{a}(u(t), \nabla u(t)) \cdot \nabla T(u(t)) d x d t \\
\leq\left\langle\mu_{S}^{T}, \phi\right\rangle+\int_{0}^{T} \int_{\mathbb{R}^{N}} \phi S(u(t)) f(u(t), \nabla T(u(t))) d x d t \\
\quad-\int_{0}^{T} \int_{\mathbb{R}^{N}} \phi f_{S}(u(t), D T(u(t))) d t \\
=\left\langle\mu_{S}^{T}, \phi\right\rangle-\int_{0}^{T} \int_{\mathbb{R}^{N}} \phi f_{S}(u(t), D T(u(t)))^{S} d t .
\end{aligned}
$$

Since the absolutely continuous part of $h_{S}(u, D T(u))$ is $S(u(t)) \mathbf{a}(u(t), \nabla u(t)) \cdot \nabla T(u(t))$ we obtain 4.27.

From the above lemma, using 4.21 we infer that the mild solution $u$ satisfies the entropy inequalities

$$
\begin{aligned}
& \int_{0}^{T} \int_{\mathbb{R}^{N}} \phi h_{S}(u, D T(u)) d t+\int_{0}^{T} \int_{\mathbb{R}^{N}} \phi h_{T}(u, D S(u)) d t \\
& \quad \leq \int_{0}^{T} \int_{\mathbb{R}^{N}} J_{T S}(u(t)) \phi^{\prime}(t) d x d t-\int_{0}^{T} \int_{\mathbb{R}^{N}} \mathbf{a}(u(t), \nabla u(t)) \cdot \nabla \phi T(u(t)) S(u(t)) d x d t .
\end{aligned}
$$

for the truncatures $S, T \in \mathcal{T}^{+}$and any smooth function $\phi$ of compact support in time, in particular of the form $\phi(t, x)=\phi_{1}(t) \rho(x), \phi_{1} \in \mathcal{D}((0, T)), \rho \in \mathcal{D}\left(\mathbb{R}^{N}\right)$. This concludes the proof of existence of solutions of 11.1 .

Uniqueness of entropy solutions. Let $b>a>2 \varepsilon>0$ and $T(r)=T_{a, b}(r)-a$. Set

$$
\begin{aligned}
R_{\varepsilon, l}(r) & :=T_{\varepsilon}(r-l)+\varepsilon=T_{l-\varepsilon, l+\varepsilon}(r)+\varepsilon-l, \\
J_{T, \varepsilon, l}(r) & :=\int_{0}^{r} T(s) R_{\varepsilon, l}(s) d s, \quad j_{T, \varepsilon, l}(r):=\int_{l}^{r} T(s) T_{\varepsilon}(s-l) d s .
\end{aligned}
$$

Let $u, \bar{u}$ be two entropy solutions of (1.1) corresponding to the initial conditions $u_{0}, \bar{u}_{0} \in$ $\left(L^{1}\left(\mathbb{R}^{N}\right) \cap L^{\infty}\left(\mathbb{R}^{N}\right)\right)^{+}$, respectively. Then there exist $\xi, \bar{\xi} \in\left(L^{1}\left(0, T, \mathrm{BV}\left(\mathbb{R}^{N}\right)_{2}\right)\right)^{*}$ such that if $\mathbf{z}(t):=\mathbf{a}(u(t), \nabla u(t))$ and $\overline{\mathbf{z}}(t):=\mathbf{a}(\bar{u}(t), \nabla \bar{u}(t))$, then we have $(\mathbf{z}(t), \xi(t)),(\overline{\mathbf{z}}(t), \bar{\xi}(t)) \in Z\left(\mathbb{R}^{N}\right)$ for almost all $t \in[0, T]$ and 
$\xi, \bar{\xi}$ are the time derivatives of $u, \bar{u}$ in $\left(L^{1}\left(0, T, \mathrm{BV}\left(\mathbb{R}^{N}\right)_{2}\right)\right)^{*}$, resp., $\xi=\operatorname{div} \mathbf{z}(t)$ and $\bar{\xi}=\operatorname{div} \overline{\mathbf{z}}(t)$ in the sense of Definition 4.3 ,

and if $l_{1}, l_{2}>\varepsilon$, then

$$
\begin{aligned}
-\int_{0}^{T} & \int_{\mathbb{R}^{N}} J_{T, \varepsilon, l_{1}}(u(t)) \eta_{t} \\
& +\int_{0}^{T} \int_{\mathbb{R}^{N}} \eta(t)\left(h_{T}\left(u(t), D R_{\varepsilon, l_{1}}(u(t))\right)+h_{R_{\varepsilon, l_{1}}}(u(t), D T(u(t)))\right) \\
& \quad+\int_{0}^{T} \int_{\mathbb{R}^{N}} \mathbf{z}(t) \cdot \nabla \eta(t) T(u(t)) R_{\varepsilon, l_{1}}(u(t)) \leq 0
\end{aligned}
$$

and

$$
\begin{aligned}
-\int_{0}^{T} & \int_{\mathbb{R}^{N}} J_{T, \varepsilon, l_{2}}(\bar{u}(t)) \eta_{t} \\
& \quad+\int_{0}^{T} \int_{\mathbb{R}^{N}} \eta(t)\left(h_{T}\left(\bar{u}(t), D R_{\varepsilon, l_{2}}(\bar{u}(t))\right)+h_{R_{\varepsilon, l_{2}}}(\bar{u}(t), D T(\bar{u}(t)))\right) \\
& \quad+\int_{0}^{T} \int_{\mathbb{R}^{N}} \overline{\mathbf{z}}(t) \cdot \nabla \eta(t) T(\bar{u}(t)) R_{\varepsilon, l_{2}}(\bar{u}(t)) \leq 0
\end{aligned}
$$

for all $\eta \in C^{\infty}\left(Q_{T}\right)$ with $\eta \geq 0, \eta(t, x)=\phi(t) \rho(x)$, where $\phi \in \mathcal{D}((0, T)), \rho \in \mathcal{D}\left(\mathbb{R}^{N}\right)$.

Now, we can rewrite (4.31) and (4.32) as

$$
\begin{aligned}
-\int_{0}^{T} \int_{\mathbb{R}^{N}} j_{T, \varepsilon, l_{1}}(u(t)) \eta_{t} & -\varepsilon \int_{0}^{T} \int_{\mathbb{R}^{N}} J_{T}(u(t)) \eta_{t} \\
+\int_{0}^{T} \int_{\mathbb{R}^{N}} \eta(t)\left(h_{T}\left(u(t), D R_{\varepsilon, l_{1}}(u(t))\right)+h_{R_{\varepsilon, l_{1}}}(u(t), D T(u(t)))\right) & \\
& +\int_{0}^{T} \int_{\mathbb{R}^{N}} \mathbf{z}(t) \cdot \nabla \eta(t) T(u(t)) R_{\varepsilon, l_{1}}(u(t)) \leq 0
\end{aligned}
$$

and

$$
\begin{aligned}
-\int_{0}^{T} \int_{\mathbb{R}^{N}} j_{T, \varepsilon, l_{2}}(\bar{u}(t)) \eta_{t} & -\varepsilon \int_{0}^{T} \int_{\mathbb{R}^{N}} J_{T}(\bar{u}(t)) \eta_{t} \\
& +\int_{0}^{T} \int_{\mathbb{R}^{N}} \eta(t)\left(h_{T}\left(\bar{u}(t), D R_{\varepsilon, l_{2}}(\bar{u}(t))\right)+h_{R_{\varepsilon, l_{2}}}(\bar{u}(t), D T(\bar{u}(t)))\right) \\
& \quad+\int_{0}^{T} \int_{\mathbb{R}^{N}} \overline{\mathbf{z}}(t) \cdot \nabla \eta(t) T(\bar{u}(t)) R_{\varepsilon, l_{2}}(\bar{u}(t)) \leq 0,
\end{aligned}
$$

We choose two different pairs of variables $(t, x),(s, y)$ and consider $u, \mathbf{z}$ as functions of $(t, x)$ and $\bar{u}, \overline{\mathbf{z}}$ as functions of $(s, y)$. Let $0 \leq \phi \in \mathcal{D}((0, T)), \rho_{m}$ a classical sequence of mollifiers in $\mathbb{R}^{N}$ and $\tilde{\rho}_{n}$ a sequence of mollifiers in $\mathbb{R}$. Define

$$
\eta_{m, n}(t, x, s, y):=\rho_{m}(x-y) \tilde{\rho}_{n}(t-s) \phi\left(\frac{t+s}{2}\right) .
$$


For $(s, y)$ fixed, if we take $l_{1}=\bar{u}(s, y)$ in 4.33 , we get

$$
\begin{gathered}
-\int_{0}^{T} \int_{\mathbb{R}^{N}} j_{T, \varepsilon, \bar{u}(s, y)}(u(t, x))\left(\eta_{m, n}\right)_{t} d x d t-\varepsilon \int_{0}^{T} \int_{\mathbb{R}^{N}} J_{T}(u(t, x))\left(\eta_{m, n}\right)_{t} d x d t \\
+\int_{0}^{T} \int_{\mathbb{R}^{N}} \eta_{m, n}\left(h_{T}\left(u(t, x), D_{x} R_{\varepsilon, \bar{u}(s, y)}(u(t, x))\right)+h_{R_{\varepsilon, \bar{u}(s, y)}}\left(u(t), D_{x} T(u(t))\right)\right) d t \\
\quad+\int_{0}^{T} \int_{\mathbb{R}^{N}} \mathbf{z}(t, x) \cdot \nabla_{x} \eta_{m, n} T(u(t, x)) R_{\varepsilon, \bar{u}(s, y)}(u(t, x)) d x d t \leq 0 .
\end{gathered}
$$

Similarly, for $(t, x)$ fixed, if we take $l_{2}=u(t, x)$ in (4.34), we get

$$
\begin{gathered}
-\int_{0}^{T} \int_{\mathbb{R}^{N}} j_{T, \varepsilon, u(t, x)}(\bar{u}(s, y))\left(\eta_{m, n}\right)_{s} d y d s-\varepsilon \int_{0}^{T} \int_{\mathbb{R}^{N}} J_{T}(\bar{u}(s, y))\left(\eta_{m, n}\right)_{s} d y d s \\
+\int_{0}^{T} \int_{\mathbb{R}^{N}} \eta_{m, n}\left(h_{T}\left(\bar{u}(s, y), D_{y} R_{\varepsilon, u(t, x)}(\bar{u}(s, y))\right)+h_{R_{\varepsilon, u(t, x)}}\left(\bar{u}(s), D_{y} T(\bar{u}(s))\right)\right) d s \\
\quad+\int_{0}^{T} \int_{\mathbb{R}^{N}} \overline{\mathbf{z}}(s, y) \cdot \nabla_{y} \eta_{m, n} T(\bar{u}(s, y)) R_{\varepsilon, u(t, x)}(\bar{u}(s, y)) d y d s \leq 0
\end{gathered}
$$

Integrating 4.35 in $(s, y), 4.36$ in $(t, x)$, adding the two inequalities, using the fact that $a>2 \varepsilon$, and taking into account that $\nabla_{x} \eta_{m, n}+\nabla_{y} \eta_{m, n}=0$, we have

$$
\begin{aligned}
-\int_{Q_{T} \times Q_{T}}\left(j_{T, \varepsilon, \bar{u}(s, y)}\right. & \left.u(t, x))\left(\eta_{m, n}\right)_{t}+j_{T, \varepsilon, u(t, x)}(\bar{u}(s, y))\left(\eta_{m, n}\right)_{s}\right) \\
& -\varepsilon \int_{Q_{T} \times Q_{T}}\left(J_{T}(u(t, x))\left(\eta_{m, n}\right)_{t}+J_{T}(\bar{u}(s, y))\left(\eta_{m, n}\right)_{s}\right) \\
& +\int_{Q_{T} \times Q_{T}} \eta_{m, n} h_{T}\left(u(t, x), D_{x} R_{\varepsilon, \bar{u}(s, y)}(u(t, x))\right) \\
& +\int_{Q_{T} \times Q_{T}} \eta_{m, n} h_{T}\left(\bar{u}(s, y), D_{y} R_{\varepsilon, u(t, x)}(\bar{u}(s, y))\right) \\
& +\int_{Q_{T} \times Q_{T}} \eta_{m, n} h_{R_{\varepsilon, \bar{u}}(s, y)}\left(u(t), D_{x} T(u(t))\right) \\
& +\int_{Q_{T} \times Q_{T}} \eta_{m, n} h_{R_{\varepsilon, u}(t, x)}\left(\bar{u}(s), D_{y} T(\bar{u}(s))\right) \\
& -\int_{Q_{T} \times Q_{T}} \overline{\mathbf{z}}(s, y) \cdot \nabla_{x} \eta_{m, n} T(\bar{u}(s, y)) R_{\varepsilon, u(t, x)}(\bar{u}(s, y)) \\
& -\int_{Q_{T} \times Q_{T}} \mathbf{z}(t, x) \cdot \nabla_{y} \eta_{m, n} T(u(t, x)) R_{\varepsilon, \bar{u}(s, y)}(u(t, x)) \leq 0 .
\end{aligned}
$$

Then, since

$$
\int_{Q_{T} \times Q_{T}} \eta_{m, n} h_{R_{\varepsilon, \bar{u}(s, y)}}\left(u(t), D_{x} T(u(t))\right) \geq 0
$$


and

$$
\int_{Q_{T} \times Q_{T}} \eta_{m, n} h_{R_{\varepsilon, u(t, x)}}\left(\bar{u}(s), D_{y} T(\bar{u}(s))\right) \geq 0,
$$

we get

$$
\begin{aligned}
-\int_{Q_{T} \times Q_{T}}\left(j_{T, \varepsilon, \bar{u}(s, y)}(u(t, x))\left(\eta_{m, n}\right)_{t}+j_{T, \varepsilon, u(t, x)}(\bar{u}(s, y))\left(\eta_{m, n}\right)_{s}\right) \\
\quad-\varepsilon \int_{Q_{T} \times Q_{T}}\left(J_{T}(u(t, x))\left(\eta_{m, n}\right)_{t}+J_{T}(\bar{u}(s, y))\left(\eta_{m, n}\right)_{s}\right) \\
\quad+\int_{Q_{T} \times Q_{T}} \eta_{m, n} h_{T}\left(u(t, x), D_{x} R_{\varepsilon, \bar{u}(s, y)}(u(t, x))\right) \\
+\int_{Q_{T} \times Q_{T}} \eta_{m, n} h_{T}\left(\bar{u}(s, y), D_{y} R_{\varepsilon, u(t, x)}(\bar{u}(s, y))\right) \\
-\int_{Q_{T} \times Q_{T}} \overline{\mathbf{z}}(s, y) \cdot \nabla_{x} \eta_{m, n} T(\bar{u}(s, y)) R_{\varepsilon, u(t, x)}(\bar{u}(s, y)) \\
-\int_{Q_{T} \times Q_{T}} \mathbf{z}(t, x) \cdot \nabla_{y} \eta_{m, n} T(u(t, x)) R_{\varepsilon, \bar{u}(s, y)}(u(t, x)) \leq 0 .
\end{aligned}
$$

Let $I_{1}, I_{2}$ be, respectively, the sum of the first two terms and the sum of the remaining terms on the left hand side of the above inequality. From now on, since $u, \mathbf{z}$ are always functions of $(t, x)$, and $\bar{u}, \overline{\mathbf{z}}$ are always functions of $(s, y)$, for brevity we shall omit the arguments except when they appear as subscripts and in some additional cases where we find it useful to recall them. Now, by Green's formula and the identities $\mathbf{z}(t, x)=$ $\mathbf{a}(u(t, x), \nabla u(t, x)), \overline{\mathbf{z}}(s, y)=\mathbf{a}(\bar{u}(s, y), \nabla \bar{u}(s, y))$, we have

$$
\begin{aligned}
I_{2}:= & \int_{Q_{T} \times Q_{T}} \eta_{m, n} h_{T}\left(u, D_{x} R_{\varepsilon, \bar{u}(s, y)}(u)\right)+\int_{Q_{T} \times Q_{T}} \eta_{m, n} h_{T}\left(\bar{u}, D_{y} R_{\varepsilon, u(t, x)}(\bar{u})\right) \\
& -\int_{Q_{T} \times Q_{T}} \overline{\mathbf{z}} \cdot \nabla_{x} \eta_{m, n} T(\bar{u}) R_{\varepsilon, u(t, x)}(\bar{u})-\int_{Q_{T} \times Q_{T}} \mathbf{z} \cdot \nabla_{y} \eta_{m, n} T(u) R_{\varepsilon, \bar{u}(s, y)}(u) \\
= & \int_{Q_{T} \times Q_{T}} \eta_{m, n} h_{T}\left(u, D_{x} R_{\varepsilon, \bar{u}(s, y)}(u)\right)-\int_{Q_{T} \times Q_{T}} \eta_{m, n} \overline{\mathbf{z}} \cdot D_{x} T_{\varepsilon}(u-\bar{u}) T(\bar{u}) \\
& +\int_{Q_{T} \times Q_{T}} \eta_{m, n} h_{T}\left(\bar{u}, D_{y} R_{\varepsilon, u(t, x)}(\bar{u})\right)-\int_{Q_{T} \times Q_{T}} \eta_{m, n} \mathbf{z} \cdot D_{y} T_{\varepsilon}(\bar{u}-u) T(u) .
\end{aligned}
$$

Let us write

$$
I_{2}=I_{2}(a c)+I_{2}(s),
$$

where $I_{2}(a c)$ contains the absolutely continuous parts of $I_{2}$, and $I_{2}(s)$ contains its singular parts. Now, working as in the proof of uniqueness of Theorem 3.6 (see [6]), we obtain

$$
\frac{1}{\varepsilon} I_{2} \geq\|\phi\|_{\infty} O(\varepsilon)
$$


Hence, by 4.37, it follows that

$$
\begin{aligned}
& -\frac{1}{\varepsilon} \int_{Q_{T} \times Q_{T}}\left(j_{T, \varepsilon, \bar{u}(s, y)}(u)\left(\eta_{m, n}\right)_{t}+j_{T, \varepsilon, u(t, x)}(\bar{u})\left(\eta_{m, n}\right)_{s}\right) \\
& \quad \leq\|\phi\|_{\infty} O(\varepsilon)+\int_{Q_{T} \times Q_{T}}\left(J_{T}(u)\left(\eta_{m, n}\right)_{t}+J_{T}(\bar{u})\left(\eta_{m, n}\right)_{s}\right) .
\end{aligned}
$$

Therefore, letting $\varepsilon \rightarrow 0$ in 4.38 we obtain

$$
\begin{aligned}
& -\int_{Q_{T} \times Q_{T}}\left(j_{T, \operatorname{sign}, \bar{u}(s, y)}(u)\left(\eta_{m, n}\right)_{t}+j_{T, \operatorname{sign}, u(t, x)}(\bar{u})\left(\eta_{m, n}\right)_{s}\right) \\
& \quad \leq \int_{Q_{T} \times Q_{T}}\left(J_{T}(u)\left(\eta_{m, n}\right)_{t}+J_{T}(\bar{u})\left(\eta_{m, n}\right)_{s}\right),
\end{aligned}
$$

where

$$
j_{T, \text { sign }, l}(r)=\int_{l}^{r} T\left(r^{\prime}\right) \operatorname{sign}_{0}\left(r^{\prime}-l\right) d r^{\prime}, \quad l \in \mathbb{R}, r \geq 0 .
$$

Now, letting $m \rightarrow \infty$ we have

$$
\begin{array}{r}
-\int_{(0, T) \times(0, T) \times \mathbb{R}^{N}}\left(j_{T, \operatorname{sign}, \bar{u}(s, x)}(u(t, x))\left(\chi_{n}\right)_{t}+j_{T, \operatorname{sign}, u(t, x)}(\bar{u}(s, x))\left(\chi_{n}\right)_{s}\right) \\
\leq \int_{(0, T) \times(0, T) \times \mathbb{R}^{N}}\left(J_{T}(u(t, x))\left(\chi_{n}\right)_{t}+J_{T}(\bar{u}(s, x))\left(\chi_{n}\right)_{s}\right),
\end{array}
$$

where

$$
\chi_{n}=\tilde{\rho}_{n}(t-s) \phi\left(\frac{t+s}{2}\right)
$$

Letting $a \rightarrow 0+$ in 4.40 we get

$$
\begin{aligned}
-\int_{(0, T) \times(0, T) \times \mathbb{R}^{N}} & \left(j_{T_{0, b}, \operatorname{sign}, \bar{u}(s, x)}(u(t, x))\left(\chi_{n}\right)_{t}+j_{T_{0, b}, \operatorname{sign}, u(t, x)}(\bar{u}(s, x))\left(\chi_{n}\right)_{s}\right) \\
\leq & \int_{(0, T) \times(0, T) \times \mathbb{R}^{N}}\left(J_{T_{0, b}}(u(t))\left(\chi_{n}\right)_{t}+J_{T_{0, b}}(\bar{u}(s))\left(\chi_{n}\right)_{s}\right)
\end{aligned}
$$

Observe that

$$
\frac{1}{b} j_{T_{0, b}, \text { sign }, l}(r) \rightarrow j(r)=|r-l| \quad \text { as } b \rightarrow 0+.
$$

Hence, dividing (4.41) by $b$ and letting $b \rightarrow 0+$, we obtain

$$
\begin{aligned}
& -\int_{(0, T) \times(0, T) \times \mathbb{R}^{N}}|u(t, x)-\bar{u}(s, x)|\left(\left(\chi_{n}\right)_{t}+\left(\chi_{n}\right)_{s}\right) \\
& \quad \leq \int_{(0, T) \times(0, T) \times \mathbb{R}^{N}}\left(u(t)\left(\chi_{n}\right)_{t}+\bar{u}(s)\left(\chi_{n}\right)_{s}\right) .
\end{aligned}
$$


Since $\chi_{n}$ has compact support in time, we have

$$
\int_{Q_{T}} u(t)\left(\chi_{n}\right)_{t}=-\int_{Q_{T}}\left\langle\xi, \chi_{n}\right\rangle=-\int_{Q_{T}}\left\langle\operatorname{div} \mathbf{z}, \chi_{n}\right\rangle=0,
$$

as $\chi_{n}$ does not depend on $x$. Similarly, we have

$$
\int_{Q_{T}} u(s)\left(\chi_{n}\right)_{s}=0
$$

Since

$$
\left(\chi_{n}\right)_{t}+\left(\chi_{n}\right)_{s}=\tilde{\rho}_{n}(t-s) \phi^{\prime}\left(\frac{t+s}{2}\right)
$$

we may write 4.42 as

$$
-\int_{(0, T) \times(0, T) \times \mathbb{R}^{N}}|u(t, x)-\bar{u}(s, x)| \tilde{\rho}_{n}(t-s) \phi^{\prime}\left(\frac{t+s}{2}\right) \leq 0 .
$$

Now, letting $n \rightarrow \infty$, we obtain

$$
-\int_{(0, T) \times \mathbb{R}^{N}}|u(t, x)-\bar{u}(t, x)| \phi^{\prime}(t) d t d x \leq 0 .
$$

Since this is true for all $0 \leq \phi \in \mathcal{D}((0, T))$, we have

$$
\frac{d}{d t} \int_{\mathbb{R}^{N}}|u(t, x)-\bar{u}(t, x)| d x \leq 0 .
$$

Hence

$$
\int_{\mathbb{R}^{N}}|u(t, x)-\bar{u}(t, x)| d x \leq \int_{\mathbb{R}^{N}}\left|u_{0}(x)-\bar{u}_{0}(x)\right| d x \quad \text { for all } t \geq 0 .
$$

This implies the uniqueness of entropy solutions. Since semigroup solutions with initial conditions in $\left(L^{1}\left(\mathbb{R}^{N}\right) \cap L^{\infty}\left(\mathbb{R}^{N}\right)\right)^{+}$are entropy solutions, it follows that entropy solutions coincide with semigroup solutions for those initial data. Then estimate 4.3 is a consequence of 3.32).

Remark 4.12. The above result will permit us to explore the qualitative behavior of solutions of the flux limited diffusion equations 3.13, 3.14), and give the evolution of the support of its solutions in a subsequent paper [7].

Remark 4.13. The convergence of resolvents described in Remark 3.10 and the characterization of semigroup solutions of $[1.1$ as entropy solutions implies that solutions of

$$
\frac{\partial u}{\partial t}=\operatorname{div} \mathbf{a}(u, D u)+\frac{1}{n} \Delta u
$$

converge as $n \rightarrow \infty$ to the entropy solution of $[1.1)$ (see [11]). 
Remark 4.14. Using similar techniques to the above ones for the Cauchy problem we may prove an existence and uniqueness result for the following Neumann problem

$$
\begin{cases}\frac{\partial u}{\partial t}=\operatorname{div} \mathbf{a}(u, D u) & \text { in } Q_{T}=(0, T) \times \Omega, \\ \frac{\partial u}{\partial \eta}=0 & \text { on } S_{T}=(0, T) \times \partial \Omega, \\ u(0, x)=u_{0}(x) & \text { in } x \in \Omega,\end{cases}
$$

where $\Omega$ is a bounded set in $\mathbb{R}^{N}$ with boundary $\partial \Omega$ of class $C^{1}, u_{0} \in L^{\infty}(\Omega)^{+}, \mathbf{a}(z, \xi)=$ $\nabla_{\xi} f(z, \xi)$, and $f$ satisfies similar assumptions to the ones considered in the Cauchy problem.

Acknowledgements. We thank José Antonio Carrillo for having attracted our attention to relativistic heat equations. We also thank Salvador Moll for several stimulating discussions concerning this paper. The first and third authors have been supported by EC through the RTN Programme Nonlinear Partial Differential Equations Describing Front Propagation and Other Singular Phenomena, HPRN-CT-2002-00274, and by PNPGC project, reference BFM2002-01145. The second author acknowledges partial support by the Departament d'Universitats, Recerca i Societat de la Informació de la Generalitat de Catalunya and by PNPGC project, reference BFM2003-02125.

\section{References}

[1] Ambrosio, L., Fusco, N., Pallara, D.: Functions of Bounded Variation and Free Discontinuity Problems. Oxford Math. Monogr., Oxford Univ. Press (2000) Zbl 0957.49001 MR 1857292

[2] Andreu, F., Caselles, V., Mazón, J. M.: Existence and uniqueness of solution for a parabolic quasilinear problem for linear growth functionals with $L^{1}$ data. Math. Ann. 322, 139-206 (2002) Zbl pre01716702 MR 1883392

[3] Andreu, F., Caselles, V., Mazón, J. M.: Parabolic Quasilinear Equations Minimizing Linear Growth Functionals. Progr. Math. 223, Birkhäuser (2004) Zbl pre02052486 MR 2033382

[4] Andreu, F., Caselles, V., Mazón, J. M.: A strongly degenerate quasilinear equation: the elliptic case. Ann. Scuola Norm. Sup. Pisa Cl. Sci. (5) 3, 555-587 (2004) MR 2099249

[5] Andreu, F., Caselles, V., Mazón, J. M.: A strongly degenerate quasilinear equation: the parabolic case. Arch. Rat. Mech. Anal. 176, 415-453 (2005)

[6] Andreu, F., Caselles, V., Mazón, J. M.: A strongly degenerate quasilinear elliptic equation. Nonlinear Anal. 61, 637-669 (2005) MR 2126618

[7] Andreu, F., Caselles, V., Mazón, J. M., Moll, S.: The speed of propagation of the support of solutions of a tempered diffusion equation. Submitted

[8] Anzellotti, G.: Pairings between measures and bounded functions and compensated compactness. Ann. Mat. Pura Appl. 4 (135), 293-318 (1983) Zbl 0572.46023 MR 0750538

[9] Bénilan, Ph., Boccardo, L., Gallouët, T., Gariepy, R., Pierre, M., Vázquez, J. L.: An $L^{1}$-theory of existence and uniqueness of solutions of nonlinear elliptic equations. Ann. Scuola Norm. Sup. Pisa (4) 22, 241-273 (1995) Zbl 0866.35037 MR 1354907

[10] Bénilan, Ph., Crandall, M. G.: Completely accretive operators. In: Semigroup Theory and Evolution Equations, Ph. Clément et al. (eds.), Dekker, 41-75 (1991) Zb1 0895.47036 MR 1164641 
[11] Bénilan, Ph., Crandall, M. G., Pazy, A.: Evolution Equations Governed by Accretive Operators. Book in preparation

[12] Bertsch, M., Dal Passo, R.: Hyperbolic phenomena in a strongly degenerate parabolic equation. Arch. Rat. Mech. Anal. 117, 349-387 (1992) Zbl 0785.35056 MR 1148213

[13] Blanc, Ph.: On the regularity of the solutions of some degenerate parabolic equations. Comm. Partial Differential Equations 18, 821-846 (1993) Zbl 0818.35050 MR 1218520

[14] Brennier, Y.: Extended Monge-Kantorovich theory. In: Optimal Transportation and Applications (Martina Franca, 2001), L. A. Caffarelli and S. Salsa (eds.), Lecture Notes in Math. 1813, Springer, 91-121 (2003) Zbl pre01984439 MR 2006306

[15] Brezis, H.: Opérateurs Maximaux Monotones. North-Holland, Amsterdam (1973) Zbl 0252.47055 MR 0348562

[16] Browder, F.: Pseudo-monotone operators and nonlinear elliptic boundary value problems on unbounded domains. Proc. Nat. Acad. Sci. USA 74, 2659-2661 (1977) Zbl 0358.35034 MR 0445124

[17] Crandall, M. G., Liggett, T. M.: Generation of semigroups of nonlinear transformations on general Banach spaces. Amer. J. Math. 93, 265-298 (1971) Zbl 0226.47038 MR 0287357

[18] Dal Maso, G.: Integral representation on $B V(\Omega)$ of $\Gamma$-limits of variational integrals. Manuscripta Math. 30, 387-416 (1980) Zbl 0435.49016 MR 0567216

[19] Dal Passo, R.: Uniqueness of the entropy solution of a strongly degenerate parabolic equation. Comm. Partial Differential Equations 18, 265-279 (1993) Zbl 0815.35056 MR 1211734

[20] De Cicco, V., Fusco, N., Verde, A.: On $L^{1}$-lower semicontinuity in $B V$. Preprint, Scuola Normale Superiore di Pisa (2003)

[21] Diestel, J., Uhl, J. J., Jr.: Vector Measures. Math. Surveys 15, Amer. Math. Soc., Providence (1977) Zbl 0369.46039 MR 0453964

[22] Duderstadt, J. J., Moses, G. A.: Inertial Confinement Fusion. Wiley (1982)

[23] Evans, L. C., Gariepy, R. F.: Measure Theory and Fine Properties of Functions. Studies in Adv. Math., CRC Press (1992) Zbl 0804.28001 MR 1158660

[24] Kruzhkov, S. N.: First order quasilinear equations in several independent variables. Math. USSR-Sb. 10, 217-243 (1970) Zbl 0215.16203 MR 0267257

[25] Mihalas, D., Mihalas, B.: Foundations of Radiation Hydrodynamics. Oxford Univ. Press (1984) Zbl 0651.76005 MR 0781346

[26] Rosenau, P.: Free energy functionals at the high gradient limit. Phys. Rev. A 41, 2227-2230 (1990)

[27] Rosenau, P.: Tempered diffusion: a transport process with propagating front and inertial delay. Phys. Rev. A 46, 7371-7374 (1992)

[28] Schwartz, L.: Fonctions mésurables et *-scalairement mésurables, mesures banachiques majorées, martingales banachiques, et propriété de Radon-Nikodým. Sém. Maurey-Schwartz, 1974-75, Ecole Polytech., Centre de Math. Zbl 0314.46041 MR 0448075

[29] Ziemer, W. P.: Weakly Differentiable Functions. Grad. Texts in Math. 120, Springer (1989) Zbl 0692.46022 MR 1014685 